Article

\title{
A Sustainable Approach to Mental Health Education: An Empirical Study Using Zhuangzi's Self-Adaptation
}

\section{Hsin-Chi Ko}

Department of Mass Communication and Language Education Centre, Chang Jung Christian University, No. 1, Changda Rd., Gueiren District, Tainan City 71101, Taiwan; hsin@mail.cjcu.edu.tw

Received: 21 February 2019; Accepted: 26 June 2019; Published: 4 July 2019

\begin{abstract}
No one can avoid feeling frustrated, and contemporary schools should take the lead in supporting mental health. A sustainable approach to such education can be found in Zhuangzi (ca 369-ca 286 BCE), a representative of Taoist schools who is credited with writing the Zhuangzi, a philosophical and literary text. The first section of this study uses qualitative research methods to identify 70 concepts regarding self-adaptation in the Zhuangzi and classifies them into 11 categories. The individual sentences from which these concepts originated are then logically reordered by category to create texts that aid a reader's understanding of Zhuangzi's philosophy. The second section of this study uses purposive sampling through an online questionnaire to consider university student feedback on self-adaptation philosophy. Overall, $84.12 \%$ of students agreed or strongly agreed that self-adaptation could help them deal with frustration, and $40.80 \%$ of students identified the category "mental state" as the most helpful. Furthermore, $88.91 \%$ of students reported that thinking about their mental state was most helpful in interpersonal relationship situations. Thus, self-adaptation offers individuals a sustainable, healthy means of dealing with life's challenges. The findings of this study may have far-reaching impacts on European and American society by cultivating the general public's interest in Zhuangzi's philosophy.
\end{abstract}

Keywords: education; mental health; health psychology; philosophy; educational philosophy; life education; affective education; modern educational issue; sustainability; Chinese curricula; Eastern philosophy; Zhuangzi; Chinese classics; Taoist regimen; philosophical therapy

\section{Introduction}

In contemporary society, the advancement of technology has brought not only convenient and comfortable lives to the public but also competition and pressure in every walk of life. When people can no longer bear the ever-increasing demands of society, their mental health suffers. We all know that one of the purposes of education is to cultivate students for socialization. Education is meant to prepare students for their future lives in society. Thus, it is important to help students become more aware of their mental health and improve their ability to make appropriate self-adjustments in the face of frustration so that they will be better equipped to handle the impacts of various situations in the challenging social environments they are going to experience.

Most educators agree that learning can be categorized based on its purpose into the following groups: Knowledge education, skills education, and affective education. Amongst them, affective education is often the weakest link even though it is also the most important issue. With knowledge and skills education, students can easily master what they are learning through repeated practice. However, affective education stands apart. Liu Shen [1] defined affection as the attitude one holds when facing an objective environment; it covers one's values and ability to adapt in life. In other words, 
affection is the mental process of facing various situations in life [1]. Therefore, affective education can be quite difficult to process and practice. As affection is subjective, the degree of difficulty a student faces in learning relevant content is also subject to individual perception.

Lin [2] suggested that affective education in contemporary universities remains incomplete. He explored the possibility of saving and improving affective education. One of his arguments for this type of education centers on the importance of developing self-awareness about life [2]. On a related note, Vincent Shen [3] discussed how affective education can help move students from the level of psychological emotions to the level of wisdom in practice, meaning students can deal with different situations they encounter while maintaining a healthy mental state. Moreover, Fong [4] argued that one of neohumanism's educational goals is to teach students internal harmony, so affective education is necessary because it is the fundamental meaning of Bildung. Obviously, in social environments filled with challenges and pressure, more attention should be paid to this perspective on types of education.

Ancient Western philosophy has helped people solve their problems psychologically. Yu [5] discussed how Socrates's (470-399 BCE) philosophical viewpoints have offered people comfort when they face difficulties in life. Epicurus (341-270 BCE) argued that philosophy can treat mental disorders and is thus good medicine for the mind. Likewise, one of the functions of ancient Chinese philosophy is to provide mental support. According to Gong [6], traditional Taoist thought contains precious wisdom on ways to keep in good health. He agreed that the book the Zhuangzi is about the philosophy of life [6]. Lee [7] argued that the Taoist school aspiration of teaching people how to manage impulsive emotions and cultivate various sentiments could induce positive mental states. Moreover, Lee [7] discussed how Master Zhu (1130-1200), the famous Chinese neo-Confucianist of Taoist origin from the Song Dynasty, proposed several philosophical treatments for better mental health including building a healthy self, increasing self-perception, enhancing willpower, and improving self-regulation, self-control, and the cognitive system. All the concepts mentioned above can be used as the academic research foundation for affective education in positive psychology.

Over time, it has been found that affective education helps improve one's ability to deal with depression. Because depression is related to personal values, many mental issues can be prevented through high-quality philosophical discussions [8]. Tun-Yi Chou was the founder of neo-Confucianism in the Song Dynasty in China, and neo-Confucianism had strong roots in Taoist philosophy, stressing that one can be comfortable and at ease when one does not perceive any psychological deficiency. Yang-Ming Wang, a Chinese psychology educator in the Ming Dynasty, proposed a similar viewpoint. He believed that one could become cheerful and overcome mental difficulties by changing one's thoughts. The potential effects of releasing repressed emotions can be found in the progression and development of Taoist philosophy. Thus, Eastern philosophy and Western philosophy have at least one thing in common: They both propose ways of dealing with repressed emotions and moving towards a healthy mind. Therefore, contemporary educators should carefully consider how they could design curricula for education based on philosophical thinking and various cultures to help students develop healthy minds and mental fortitude.

Shu [9] suggested that Taoist philosophy has had amazing educational influences on university students and a very broad impact on modern society. Outside of Asia, Taoist philosophy has also been enthusiastically studied in Europe (especially in France and Germany) and the United States [10]. In 2017, the Laozi Institute was founded in New York. It is a university that promotes the inclusion of classic Taoist works in teaching materials for mainstream public and private schools in the United States; plans campaigns at the primary, secondary, and higher education levels; and facilitates cultural and academic exchanges between the West and the East across the United States [11]. The reason this trend of learning about Taoist culture was formed in the United States is because Taoist philosophy is perceived as being very popular, or in other words, closely related to people's daily lives. The general public in the United States believes that Taoist philosophy is helpful on a personal level [12]. This fact supports the relevance of Taoist philosophy in the age of advanced technology. 
As is, the rapid development of science and technology has not only led to highly convenient lifestyles in society but also contributed to many problems in people's lives. In other words, people today are endlessly facing the unintended impacts and challenges of various innovations. Once they meet difficulties that can only be overcome by changing their mindset, something most people struggle to do, their problems may end in tragedy [13]. This type of outcome is not reserved to a small number of individual cases. Tragedies of this sort can be found all over the world all the time [14]. Therefore, many scholars have proposed solutions for the issues commonly seen in modern society. For example, one study used the lens of education for research guided by the belief that life education is inseparable from our daily existence and the personal issues we face; thus, societal problems should be solved through education [15]. Another study employed a philosophical standpoint to discuss how we can live better lives through our perceptions of the environment and the society we exist in [16]. Many scholars today feel that solving such societal problems through a philosophy of life is an important and worthy cause.

Bernard Li [17] suggested that ancient Chinese philosophies of life covering topics from individual accomplishments to harmonic relationships between humans, between humans and society, and between humans and nature are all based on the spirits of noble characters who show people how to achieve human dignity and strength. More specifically, Wei-Dong Li [18] argued that Zhuangzi's philosophy is filled with moral aesthetics for survival and reflected that Zhuangzi emphasizes valuing life and protecting and respecting individual lives in hopes that people will be able to find a way out of their own heads and will instead seek spiritual freedom without being obstructed by societal factors.

Moreover, studies have also been done using human minds as the foundation for solutions. Chen [19] suggested that the issue of how people's minds interact with and make sense of the world has been troubling philosophers for over three hundred years. Vincent Shen [20] also argued that Zhuangzi focuses on internal practices in order to develop the real self beyond the physical self. These practices provide individuals with a way to keep from seeing external people and things through a narrow perspective. By surpassing such a restriction, people are less likely to become anxious and struggle before ultimately ending up in a deadlock [21]. On the contrary, Zhuangzi believes that one should replace one's own bigoted opinions with a magnanimous attitude so that one's mind can exist in a settled state [22,23]. Based on all these scholars' views, spiritual self-cultivation of the mind is a very important aspect of Zhuangzi's philosophy.

Zhao-Hui Huang [24] further stated that over the past one thousand years, Eastern and Western philosophers have been searching for solutions to difficult life problems throughout their careers. Laozi's and Zhuangzi's ideas form a complete philosophical system with far-reaching implications. This system can heal negative thinking and provides knowledge for all kinds of issues in life [24]. People's daily struggles and these past scholars' comments regarding the positive impact Zhuangzi's ideas can have on modern society are the reasons why this study was proposed to further illuminate Zhuangzi's philosophy of life, which encourages a mind free from worldly care. As Wung-Hong Huang [25] has argued, cognitive activities are intentional experiences, so through cognition and the cultivation of reason, human minds can be elevated to overcome all kinds of difficulties encountered in life. Therefore, Zhuangzi's philosophy can help sustain human existence. The research background, research motivations, and purposes of this study are detailed in the upcoming sections.

One of the most famous scenes from the book the Zhuangzi in the Chinese world is the following conversation:

Zhuangzi and Huizi were talking on the Hao River Bridge. Zhuangzi said, "The fish are swimming around, feeling free and unrestrained. This is a fish's happiness."

Huizi said, "You are not a fish. How do you know if the fish are happy?"

Zhuangzi replied, "You are not me. How do you know that I don't know if the fish are happy?"

Huizi said, "I am not you, so I don't know what you know. However, you are not a fish, so you definitely don't know if the fish are happy." 
Zhuangzi answered, "Let's go back to the root of the question. At first, you asked, 'How do you know if the fish are happy?' You asked this based on the fact that you know that I know the fish are happy. Now what I can tell you is that I didn't know they were happy until I walked by the Hao River."

The above dialogue between Zhuangzi and Huizi, which can also be called a debate, is widely known in the Chinese literary field. Primary and secondary schools often include this dialogue in textbooks, and universities often include it in their Chinese curricula. For novices or readers outside the classroom, it is simply an interesting exchange. If the text is only being taught in terms of interpretation and the translation of the meaning of classical Chinese, a great deal will obviously be lost, because the essence of this debate does not lie in the meaning of the words but in the state of mind of the speakers. Therefore, this study's deeper analysis aims to clarify Zhuangzi's theoretical outlook so that students can have a deeper understanding as they are learning. At the same time, this study also provides teachers with in-depth information to make it easier for students to learn about the philosophy of Zhuangzi, which will help cultivate student growth and wisdom.

The researcher discovered the meaning behind these words when studying the Zhuangzi and found the conversation to be more than just interesting, as it is based on the essence of self-adaptation proposed by this study. Self-adaptation yields a carefree mental state that results in happiness. To interpret the conversation above based on this view, the author further discovered that the root of happiness according to Zhuangzi is this macro view of the world. Thus, this study's innovative exploration of Zhuangzi's idea of self-adaptation provides a way to share the essence of happiness to many readers around the world without the constraints associated with the difficult text of the Zhuangzi. Achieving happiness is the meaning and value of studying self-adaptation.

Zhuangzi (ca 369-ca 286 BCE) was born in Song, now Shangqiu City, during the Warring States period (ca 471-ca $221 \mathrm{BCE}$ ) and given the first name of Chou. During this time, the states were in turmoil, and because many people were poor, Zhuangzi developed a sympathetic understanding of life. The Zhuangzi, the book that bears his name, is a philosophical work, and the version most often read today is a revision by Syang Gwo (ca 252-312) with main chapters (33 total) divided into subchapters (seven inner and 15 outer) and other various chapters (11 total). The book contains original ideas about life philosophy that are presented in fable form: Stories, words, or descriptions of events are used to express the writer's own thoughts, emotions, and arguments. The fables in the Zhuangzi are critically sarcastic, particularly regarding the prevailing social order and culture, and they reveal Zhuangzi's antipathy towards the times in which he lived. The fables also elaborate on how to cultivate a tolerant spirit for suffering and how to transcend demanding situations to achieve a mental state that exists beyond life and death—spiritual freedom—even though the body is restrained by its corporeal nature. This is a psychological liberty, a life philosophy through which the practitioner rises above worldly issues. The Zhuangzi, acclaimed in both the Tsin dynasty (265-420) and the Tang dynasty (618-907), profoundly influenced both cultures, and its author is now celebrated as a leading philosopher and writer of his time. Since his influence is felt even today, this study proposes that Zhuangzi's philosophy is related to a broader life philosophy and that his ideas comprise a comprehensive humanistic view of the world.

To help readers understand the content of the Zhuangzi, this study summarizes the central ideas of the book's 33 chapters, thus covering the seven subchapters (1-7), 15 exoteric chapters (8-22), and the 11 other various chapters (23-33) [26].

Chapter 1, "Carefree Wandering", is perhaps the book's most important chapter. The central argument is that different attitudes towards life are based on different perspectives, which Zhuangzi illustrates using a rhetorical technique by contrasting a large bird and a small one. In Chapter 2, "On the Equality of Things", Zhuangzi elaborates on the idea that all earthly things are uniform. Here, the key idea is to combine the individual self and the greater self to achieve a state of uniformity and inspire a spirit of transcendence. In Chapter 3, "The Way of Nurturing Life", Zhuangzi describes the principles of a nurturing life; that is, working with nature allows people to attain psychological well-being beyond the states of honor and disgrace or earthly gain. 
Chapter 4, "In the Human World", is chiefly concerned with sustaining interpersonal relationships in a turbulent society, and Chapter 5, "Symbols of Abundant Morals", elaborates on this same topic. Here, Zhuangzi introduces life's most important lesson: The perfection of the mind. He considers human life from two aspects- the physical body and the human mind-using fables to explain how mental cultivation can transcend the body to reach a higher state of being. Similarly, in Chapter 6, "Great Master", Zhuangzi is concerned with the relationship between nature and humankind. He explains that to understand life, we must learn from the laws of nature, which reflect an ordered universe.

In Chapter 7, "Responding to Kings", Zhuangzi discusses why politicians should govern in accordance with nature's laws, and in Chapter 8, "Toes Connected", he explains that men should comply with their nature to deal with personal and interpersonal relationships. Acting according to their nature, however, does not mean that they should pursue fame, material advantages, or other worldly ambitions. Thus, in Chapter 9, "Hooves of Horses", Zhuangzi introduces the political ideal of governing in a manner that respects nature and asserts that people should live their lives according to natural laws. For this reason, political leaders should practice benevolence and righteousness, thereby minimizing the social chaos that results from ambition, fame, and wealth.

Chapter 10, "Open the Box to Steal Things", teaches that politicians should lead by example and help people return to lives of simplicity. In fact, the principal argument of Chapter 11, "Noninterference", is that if political leaders can achieve self-cultivation of peace and inaction, they will become role models who motivate others to build a harmonious society. Although all things on earth follow natural laws, Zhuangzi reminds the reader in Chapter 12, "Heaven and Earth", that people often violate these laws. Chapter 13, "The Principle of Heaven", discusses social behavior based on the laws of nature. Zhuangzi explains in Chapter 14, "The Evolution of Heaven", how changes in nature multiply without end. However, people should always adapt, constantly reconciling themselves to their situations in the world. To this end, Chapter 15, "Strengthening the Will", analyses six attitudes of living and exalts the personality that transcends honor and disgrace, cost and gain.

Chapter 16, "Mending the Nature", talks about how to cultivate a moral character amid suffering and misery. In Chapter 17, "Floods of Autumn", Zhuangzi examines social issues, using imaginative comparisons to support his points. The main argument of Chapter 18, "Topmost Happiness", defines the highest state of happiness, to which we should all aspire. Chapter 19, "Comfortable Life", is principally about maintaining our well-being in a complex environment. This chapter explains how we can avoid being influenced by the environment and maintain an ideal state of mental peace. Thus, Chapter 20, "Mountain Tree", focuses on how to avoid hazards while living in a perilous world. Chapter 21, "Tyan Dz Fang", teaches that success in life does not depend on fame or fortune. True success begins with freeing oneself from counterfeit goals, which places us on the real path to freedom. In Chapter 22, "Travel to the North", Zhuangzi explains why all earthly things follow nature's course and that life's major principles can only be learned by following nature's example. Chapter 23, “Geng Sang $\mathrm{Chu}^{\prime \prime}$, uses the metaphor of a baby without worry to illustrate that the way to achieve a peaceful mind is to eliminate personal desire. Lawlessness and punishment are the result of desire and its subsequent obsessions.

In Chapter 24, "Syu Wugwei", Zhuangzi maintains that the key to harmony for people, societies, and countries is the principle of self-cultivation of peace and inaction. Thus, Chapter 25, "Dze Yang", reminds us to reflect on our lives according to the laws of nature and to spurn the pursuit of fame and fortune. Chapter 26, "External Things", focuses on the necessity of elevating our psychological levels to understand that one of humanity's failings is the use of knowledge for immoral purposes. Chapter 27, "Implied Story", explains that people should abandon the idea of plotting against one another. We should transcend adverse circumstances and instead show our pure nature. Chapter 28, "Yielding the King", explains that health and morality are far more important than fame and fortune. Chapter 29, "Robber Jr", suggests that men should follow their true nature: There is no need to pursue fame or fortune under the guise of benevolence and righteousness. In truth, men should seek humanity and a culture of simplicity. 
Chapter 30, "Discussion on Swords", talks about the consequences of a country becoming poorer if its lord is addicted to swordsmanship (i.e., the martial arts) and ignores sound governance. Chapter 31, "The Old Fisherman", identifies humankind's common mistakes and emphasizes the idea of self-cultivation of peace and inaction. Chapter 32, "Lye Yukou", promotes the key point of maintaining a peaceful life and good health, and of opposing the pursuit of fame and fortune, which is motivated by vanity, arrogance, and hypocrisy. Finally, Chapter 33, "The World", reviews important academic figures in the pre-Chin period (ca $221 \mathrm{BCE}$ ) and draws a general conclusion for the entire work.

Based on this summary of the book's 33 chapters, it is clear that the Zhuangzi emphasizes the idea of self-adaptation, that is, a concept of self-happiness-oriented learning. The proceeding analysis is based on Zhuangzi's philosophy.

Since Zhuangzi is an important representative figure of Taoism, there have been many studies related to the Zhuangzi text and the author himself. In recent years, scholars have become increasingly interested in the Zhuangzi because there have been, on the one hand, new discoveries regarding the text and, on the other hand, a recognition that its ideas are relevant in the modern age. To help readers understand these ideas and the context of the research being presented, the literature review has been split into eight categories of research directions, which are summarized below.

Category 1 covers studies regarding only one article from the book. Examples include (a) a study about the organizational structure and expressions of "Mountain Tree" [27], (b) a study analyzing life's demanding situations in "On the Equality of Things" [28], and (c) a study analyzing the spirit of the Zhuangzi based on the fables found in "Carefree Wandering" [29].

Category 2 covers any studies that discuss the philosophical ideas of the Zhuangzi. Examples include (a) a study analyzing the relationship between philosophy and imagery in the Zhuangzi [30], (b) a study on new research methods for Zhuangzi's theory of human nature (including the pre-theory period research method and the category theory research method [31], (c) a study discussing Zhuangzi's ideas about tolerance [32], (d) a study analyzing Zhuangzi's philosophy by delving deep into his wisdom [33], (e) a study analyzing Zhuangzi's ideal goals pertaining to the subjective spirit [34], (f) a study analyzing the influence of Zhuangzi's philosophy on travel and enlightenment [35], (g) a study analyzing Zhuangzi's views on the importance of good health and its implications for a good life [36], and (h) a study discussing the meaning of the concept of "useless use" [37].

Category 3 covers studies pertaining to the correction of certain terms in the Zhuangzi. Examples include (a) a study analyzing the philosophy of life in Zhuangzi's use of the word fish to symbolize people [38], (b) a study correcting the meaning of the word ruthless as used by Zhuangzi [39], (c) a study clarifying the different meanings of the word wandering and exploring the meaning of Tao according to Zhuangzi [40], (d) a study analyzing the meaning of the word wander in the Zhuangzi [41], (e) a study analyzing the concept of chi in Zhuangzi's philosophy [42], and (f) a study about the relationship between the words use and Tao [43].

Category 4 covers studies that review ideas about the Zhuangzi. Examples include (a) a study reinterpreting the Zhuangzi from a unique critical viewpoint [44], (b) a study analyzing Ju-Syi's agreement with Zhuangzi in the description of Tao as reality and its limitations [45], and (c) a study analyzing Lyu's interpretation of the purpose of the Zhuangzi and topics on anatta, a Buddhist doctrine about the "non-self" [46].

Category 5 covers studies exploring the viewpoint of the material body. Examples include (a) a study analyzing the metaphors used in the Zhuangzi for descriptions of and reflections on death [47], (b) a study analyzing Zhuangzi's attitudes towards life and death [48], (c) a study exploring the sensual experiences and physical practices in the Zhuangzi [49], (d) a study exploring self-transformation issues involving the body and metaphors [50], (e) a study constructing the phenomenology of chi based on Zhuangzi's ideas about the body [51], and (f) a study exploring the implications of the perception of communication proposed by Zhuangzi [52]. 
Category 6 covers studies that take a psychological view of the concept of Tao in the Zhuangzi. Examples include (a) a study using Tao as the core idea of the Zhuangzi [53] and (b) a study analyzing the deeper meanings of the subjective and objective aspects of a Tao mind [54].

Category 7 covers studies comparing the ideas presented in the Zhuangzi with other theories. Examples include (a) a study investigating the relationship between the Zhuangzi and the study of Confucian classics based principally on the seven inner chapters of the Zhuangzi [55], (b) a study discussing the differences in ideas between the Zhuangzi and the Mohist School [56], and (c) a study on the words used in the Zhuangzi that are similar to those used by the Yin-Yang School [57].

Category 8 covers studies that analyze the Zhuangzi's ideas concerning literature and art. Examples include (a) a study on the ideas about the beauty of art and nature found in the theories of the Zhuangzi [58], (b) a study disclosing the theoretical contributions of Zhuangzi's doctrines to the literature in the Song Dynasty [59], and (c) a study analyzing the association between emptiness and stillness and active literary creation [60].

Of the above eight categories, Category 3 is the largest and has the most studies. There are several important terms in the Zhuangzi that reflect philosophical thoughts. The directions of research for the studies in Category 1 and Category 7 are common. Meanwhile, the topics of the studies in Category 5 are relatively new. In recent years, there has been considerable research conducted that is meant to highlight applications to issues in modern life. It is certainly true that in advanced societies, pressures from life and work are often burdensome. For this reason, this study focuses on the idea of self-adaptation, which is concerned with psychological and spiritual transcendence. This concept not only includes issues of life philosophy regarding psychological adjustment to modern life but also facilitates the application of academic research findings to daily life.

\subsection{Problem Statement}

Briefly, the Zhuangzi deals with the internal burdens and social pressures that we are all subject to and suggests a path leading to spiritual and psychological enlightenment. Being physically restricted to the external, objective environment compels us to focus on our psychological well-being, thus creating a transcendent force of the psychological self that originates deep inside each of us. This force helps us to manage and even overcome the inevitable sorrows and disappointments of life. The Zhuangzi contains not only profound insights into education - the meaning of learning, enlightenment, moral restraint, and manners-but also a blueprint for a positive, happiness-based philosophy.

Zhuangzi believed that living means coping with life and that we would quickly lose faith if we could not bear life's burdens. The way to transcend these burdens is to manifest our God-given abilities and strengthen our will to overcome worldly concerns. Although this ability to self-adapt-the focus of this study-is rooted in nature, Zhuangzi believed that nature itself is unlimited and that its characteristics include order and stable evolution. We must, therefore, ask ourselves, what can we learn from nature? The Zhuangzi answers this question by saying that we should see things from the height of a roc (a legendary bird of prey), meaning that we should broaden our horizons, going beyond the immediacy of things, and be open-minded and magnanimous. Achieving such perspective improves our psychological level, a step on the path to being carefree.

\subsection{Purpose of the Study}

The purpose of this study is to explore the concept of self-adaptation proposed by Zhuangzi in his eponymous text, the Zhuangzi. The book contains 33 chapters, categorized into inner, outer, and miscellaneous chapters, and consists of about 65,000 words. The study employs a content analysis, categorizing and synthesizing concepts related to self-adaptation to identify comprehensive content for readers who might find reading ancient Chinese classical literature difficult.

\subsection{Research Questions}

The study seeks answers to the following research questions: 
1. How many concepts related to self-adaptation appear in the seven inner chapters?

2. How many concepts related to self-adaptation appear in the 15 outer chapters?

3. How many concepts related to self-adaptation appear in the 11 miscellaneous chapters?

4. How can the concepts related to self-adaptation from the Zhuangzi be categorized?

5. What do university students think about these concepts and which are the most helpful for self-adaptation?

\subsection{Significance of the Study}

The fundamental concept behind Zhuangzi's discourse on learning from nature is the self-expectation of evolving to be magnanimous in mind and feel pain internally due to external factors. This concept is the essence of self-adaptation; it is not only the core of the Zhuangzi philosophy but also a critical issue in life philosophy in general and a positive life-affirming learning goal. Therefore, this study explores the issue of self-happiness-oriented learning, which is based on the concept of self-adaptation.

At the heart of Zhuangzi's philosophy is the notion of self-adaptation, a concept as relevant and meaningful now as it was 2500 years ago. Faced with mounting challenges and frustrations, people today need conceptual tools to strengthen the positive elements of their lives and transform the negative. By drawing on Zhuangzi's thoughts, this study presents his idea of self-adaptation, a method of preventive philosophical thinking that turns negative outlooks into positive ones and turns self-absorption into self-elevation.

The study's main contribution is to revisit Zhuangzi's philosophy and transfer it from the ancient East to the modern world, where people judge the value of their lives primarily based on their career paths instead of, as Zhuangzi believed, valuing life for its own sake. To be clear, comprehending and applying the philosophy of self-adaptation from the Zhuangzi means learning to deal with life's frustrations and disappointments while retaining a peaceful state of mind. In terms of life philosophy, this concept is not only an innovative learning topic but also a confirmation of the value of life.

The author has spent time observing and paying attention to educational phenomena. Therefore, the literature review explores and includes studies from 1998 onwards. This study also contributes to the literature by motivating people to focus on the aspects of self-education found in Zhuangzi's philosophy, thus helping people better adapt to life's trying situations. If the purpose of philosophy is to solve problems, then the value of Zhuangzi's philosophy is its ability to offer solutions to the many ethical and practical challenges people face every day.

\section{Method}

The research work involved an empirical study using both qualitative and quantitative research methods [61]. The study adapted purposive sampling for the quantitative research method based on the essence and goal of this research work and many scholars' perspectives. Therefore, this study contained two sections. The content analysis method was applied in the first section. Qualitative analyses were conducted based on the concept of self-adaptation from the Zhuangzi. Then the content related to self-adaptation was categorized. The aim in the second section of the study was to collect university students' opinions using purposive sampling [62]. It should be noted that the meaning of the word mental in the study's title was based on the dictionary definition of the word mind [63-67]. It has nothing to do with intelligence or brains.

\subsection{The Qualitative Research Method}

To explore the concept of self-adaptation in the Zhuangzi, the first section of this study adopted content analysis and concept analysis methods, both of which are qualitative research methods [68]. The term self-adapting can be found in two articles in the text. It first appears in the inner chapter "Great Master" and then shows up a second time in the eighth outer chapter, "Toes Connected". 
The meaning of self-adaptation in these two places shares a common spirit and emphasizes that the most important consideration is the preservation of one's original nature. In Chinese, the term self-adapting is composed of two characters: $z i$ (Chinese pinyin) [69], meaning self, and shi (Chinese pinyin) [70], meaning comfortable. Although the meaning of $z i$ is clear, it is necessary to explain the meaning of shi in detail so that the reader can thoroughly understand this core term.

According to the Revised Online Chinese Dictionary published by the Ministry of Education, shi, an adjective, is defined as being comfortable and contented [71]. In Lin Yutang's Chinese-English Dictionary of Modern Usage, shi is defined as being "comfortable, free and easy, contended" [72] (p. 835). In the Far East Chinese English Dictionary by Liang Shih-Chiu, shi is defined as being "comfortable" and "at ease with oneself" [73] (p. 1534). The common words these definitions share are comfortable and easy or at ease, with associations of being free and with oneself. According to these interpretations then, it is clear that being self-adapting means being comfortable and at ease with oneself. Thus, this study examines the implications of self-adaptation and not just the method. In other words, the study explores the content of self-adaptation in relation to pertinent concepts presented in the 33 chapters of the Zhuangzi. Given that the subject of this study was the text of the Zhuangzi itself [26], the study did not examine whether the book was truly written by Zhuangzi the philosopher.

The research steps were as follows: (a) Read the textual content, (b) mark any content associated with self-adaptation, (c) refer to any corresponding annotations, (d) refer to any annotations made by other scholars, (e) consider the compiler's views, (f) compare all of the collected content, (g) translate the classical Chinese into vernacular Chinese, (h) confirm the original text and its sources, (i) confirm the meaning of the vernacular Chinese translations and record them if no errors are found, (j) summarize all the recorded content, $(\mathrm{k})$ categorize the content, $(\mathrm{l})$ check and select suitable content, and $(\mathrm{m})$ for content in the same category, adjust the order as needed to create an easy-to-read passage based on logic. The guiding principle is to select any content with clear implications for self-adaptation and translate it from Chinese to English using Yale Pinyin. The purpose of this work was to correct the common misconception that the Zhuangzi is abstruse and unfathomable. It is hoped that this study would offer readers a chance to learn Zhuangzi's philosophy of self-adaptation without running into difficulties with reading the text.

Thus, in the research process, the study did not include any content that was related to difficult concepts. The rules determining the suitability of content were as follows:

(a) Content was not included that was too abstract to explain using specific definitions. For example, in Chapter 4, "In the Human World", Zhuangzi says that there are two codes of conduct that everyone must follow: The mandate of Heaven, and morality and justice. The concept of a mandate from Heaven is too abstract, and therefore was omitted.

(b) Content was not included that had a social ethos rooted in its particular time and place and could not be generalized to modern times. For example, in Chapter 23, "Geng Sang Chu", several sentences state that human hearts are more dangerous than mountains and rivers. Knowing people is more difficult than knowing Heaven. In nature, there is still the regular four seasons, as well as day and night. When it comes to humans, it is impossible to see through their surfaces and know their minds. These sentences also comment on how humans can do more harm than weapons.

(c) If different pieces of content convey the same concept, only one was selected in order to avoid repetition. For example, in Chapter 4, "In the Human World", there are two paragraphs that stress the same concept. The first says that big trees cannot be made into wood because their texture is too soft, which is why they should not be chopped down. The second paragraph says that those who are different from normal people on the outside should not be recruited by the army. Without any hindrance in mind, these are individuals who get to live long lives. Since these two paragraphs express the same concept, only one has been selected for analysis.

(d) Metaphysical content was too difficult to comprehend, so it was therefore not included. For example, Chapter 1, "Carefree Wandering", maintains that external things cannot cause harm, floods and deluges cannot cause drowning, and even droughts that can melt metals and stones and 
can scorch mountains and dirt cannot make someone feel hot. These types of statements require too much interpretation and may be difficult for readers to understand.

(e) Content was not included that was based on obscure guiding principles. For example, in Chapter 4, "In the Human World", Zhuangzi asks readers if they know the trick of raising a tiger. It cannot be fed with animals that are not yet dead; otherwise, its killer nature may be triggered when it bites the animals. The trick is to know when it is hungry and not to incur its wrath. Even though tigers are not the same kind of animals as humans, they can still be flattered by those who feed them. The knowledge presented here is not something that would be classified as a rule of thumb.

(f) Content that conveys concepts indirectly was not included. For example, in Chapters 2 and 4, "On the Equality of Things" and "In the Human World" respectively, there are numerous indirect expressions. The phrases "two sides of the same coin" and "being subjective may lead to an adverse effect" are both expressions with implied meanings that require deeper interpretation.

(g) Content with exaggerated expressions was not included. For example, in Chapter 32, "Lye Yukou", a sentence states that "heaven and earth are used as coffins, the sun and the moon are used as two jade disks, stars are used as gems, and all things are used as grave goods buried with the dead". All of these metaphors deal with abstract concepts that are far removed from the human experience.

(h) Content that requires specific associations in order to understand symbolic meanings was not included. For example, there is a paragraph in Chapter 1, "Carefree Wandering", that says that rocs (a legendary bird of prey) are birds with huge bodies. There are some small birds called zhens, which are legendary birds with poisonous feathers. When they see rocs fly high in the sky, they laugh at them: "We little birds can take off and land whenever we want, unlike big birds like you that can only take off with the wind. Furthermore, little birds like us can fly to the trees or to the ground as we please. 'Why do we need to fly so high at 45,000 km?'” The fact that the meaning of this passage relies on knowledge about birds means that it can be difficult for readers to understand and relate to.

(i) Content was not included that describes troubles in the world that were indirectly related to the concept of self-adaptation. For example, a passage in Chapter 33, "The Old Fisherman", says that Confucius asked an old fisherman for advice. In response, the fisherman described the eight flaws and four troubles that are worthy of investigation. The eight flaws include boasting of one's quarrels to others, taking on everything, insidiousness, flattering, toadying, slandering, causing harm to another, and wickedness, and the four troubles include wantonness, greed, stubbornness, and arrogance. Since these troubles do not have clearly stated ties with self-adaptation, they were omitted.

The main purpose of these nine rules was to determine which pieces of content were too difficult to understand and therefore should be excluded. These rules were also this study's research limitations. Thus, the guiding principle of the research method used in this study to explore the idea of self-adaptation in the Zhuangzi was to make sure that the resulting content was easy to read and understand so that the academic findings could be broadly applied, thus increasing the value of the academic research.

\subsection{Purposive Sampling as a Research Method}

Many scholars' opinions were reviewed before purposive sampling was adopted as the quantitative research method in this study. Given that this study crosses many different academic disciplines, opinions were gathered from scholars in fields such as philosophy, literature, education, and the sciences.

Numerous relationships exist between science, philosophy, and education. Chang [74] suggested that research through sensory media belongs to the epistemological approach towards science. Wu [75] argued that research on the nature, functions, and purposes of education from the perspectives of philosophy or other fields could be classified as research on educational phenomena. Through the eyes of science, pedagogy can be interpreted as an applied social science, and it is associated with both science and sociology. Some scholars have even called it a normative science [76]. Popkewitz [77] believed that there are three major sects of educational science research. This study features empirical, 
symbolic, and linguistic considerations, which are related to the three research aspects of educational science. Based on the core linguistic research completed on Zhuangzi's concept of self-adaptation, this study identifies the educational functions of good mental health. Then this study conducts a survey of university students and performs related analyses regarding mental health experiences through the lens of Zhuangzi's concept of self-adaptation. As educational research itself focuses on value-related issues, this study fits various scholars' definitions of educational phenomenon research and thus is a work of normative science.

Integrating studies from different academic fields helps broaden and deepen connections made in academic contexts. It is hoped that the range of applications for the study's research findings can be expanded in this way. This study presents research on two key topics in the field of social science, namely, self-adaptation and mental health. This interdisciplinary study is informed by disciplines such as philosophy, literature, pedagogy, sociology, psychology, counseling, educational philosophy, educational psychology, and educational sociology. Although there have been a few studies on self-adaptation, there have not been any studies discussing these two closely related topics. In other words, to the author's knowledge, this study is the first of its kind to explore the educational functions of Zhuangzi's concept of self-adaptation in relation to mental health from an interdisciplinary perspective. The first phase of this study explores the concept of self-adaptation and shares a theoretical conclusion on the topic. The second phase uses an online questionnaire to identify the most suitable method to maintain mental health. Thus, this study is a pioneering work as well as an exploratory one. By summarizing numerous scholars' viewpoints on research theories, this study builds a solid academic foundation for educational theories.

In July 2002, the United Nations conducted a global survey, and Dai [78] identified from this survey that people from different regions and cultures shared the common problem of struggling to understand the text in the questionnaire. This survey was seen as a big failure [78]. Using this case as an example, we could identify the factors that resulted in the survey's failure by looking at various scholars' viewpoints. $\mathrm{Wu}$ [75] suggested that in comparison to natural science research, social science research is more complex in terms of the factors involved. As a result, findings are often less generalized and contain larger errors. These disadvantages can also be found in educational research, and more attention should be paid to them. Thus, $\mathrm{Wu}$ [75] believed that to obtain more accurate research results, one must find the most suitable research method for a specific research topic. Chang [74] has argued that social scientists play an important role in helping survey subjects immerse themselves in research scenarios based on the specific research topic. The only way to make a study valuable is to face the research question in a thoughtful manner so that the research findings will be based on realistic viewpoints. More importantly, the most suitable research method should be selected based on the properties of the unique research topic [74].

The research subjects of social science studies are largely influenced by human nature and social systems [79]. In the field of pedagogy, research must be conducted from a human-centered view because one of the fundamental elements of society is humans and human development is associated with education development. This is why the individualization of education is important. Social changes may also influence individual development. Therefore, it is essential to respond accordingly to the evolution of society or to prevent the negative influences of various societal phenomena through education. Thus, from the microcosmic view, this study agrees with the argument proposed by Chang and Tan. Chang and Tan [80] indicated in their book The Discussions and Applications of Educational Research Methods that pedagogy is a social science, and therefore it should focus on special and individual phenomena as idiographic science does. From the macroscopic view, this study agrees with Chen's [81] suggestion that educational and academic development should occur in response to the demands that arise from social changes.

The viewpoint proposed by Chang and Tan applies to qualified research. Kao's book Qualified Research Methods contains further insights relevant to education. He analyzed and summarized the viewpoints of educational research scholars and argued that in the face of complex educational 
phenomena, the focus should be on interpretive understanding of the phenomena. The purpose of conducting qualitative research is to explore and discover new questions and to interpret perceptions, meanings, and values in daily situations in order to learn more about humans, develop theories, improve facilities, and build foundations for theories. Qualitative research makes it possible to obtain real, deep, and credible pieces of information using humans' perceptions in natural situations [75]. The process of studying an educational phenomenon covers exploration and practice with real educational values and can be used in the development of a theory. The first and second phases of this study are both exploratory. The second phase in particular is focused on the transition from exploration to theory development. This study decided to adopt qualitative research methods because of the various considerations mentioned above.

This study was an exploratory work. Six scholars have suggested adopting the purposive sampling method in cases of exploratory research. Exploratory research is about illustrating or reviewing a topic of interest that has not yet been discussed in previous studies, and exploratory research is completed in hopes of coming up with new viewpoints or building foundations for future hypothesis testing [74]. Huang and Jiang [82] indicated that purposive sampling is important for exploratory research and can be used to pave the way for more sophisticated studies in the future. The following three scholars share the viewpoint that exploratory research is a starting place for future work. Wang and Wang [83] argued that purposive sampling is suitable for surveys on people's attitudes or thoughts. However, it has its limitations [83]. Chuang found that purposive sampling provides meanings based on the specific context of the research topic. That is, when this sampling method is applied, the representativeness of the sample can be ignored. Purposive sampling focuses on applications and makes it possible to select a sample with an exemplary meaning in theory. The representativeness of the sample is not the priority in such a study. These three scholars believed that purposive sampling in the initial research phase has the advantage of making it possible to explore exemplariness in theory through a survey and the disadvantage of possibly low representativeness.

Regarding the representativeness of a sample, some scholars have proposed a critical approach. Lin [84] suggested that researchers could adopt an intentional sampling method based on their understanding of the target population, the research objective, and their subjective judgments so that they can quickly retrieve useful information from the subjects' responses to the questions. This concept is commonly described as purposive sampling. Lin [84] further indicated that this sample might not be representative. Although it cannot represent the population, it is sufficient to provide reliable information. Lin [84] believed that the key is to make sure the subjects will not resist the research process due to their unfamiliarity with the topic, an act that would result in the failure of the research. When a subject is not willing to participate or has other considerations in mind, Lin [84] said that the research itself is likely to yield errors. This puts exploratory research in a vulnerable position. If the subjects' attitudes are not carefully evaluated, the results of a questionnaire may be biased in cases where a large percentage of the subjects refuse to accept new concepts. In more serious cases, possible contributions to education made based on the theory of the research may be destroyed. With this analysis, it is clear that purposive sampling is related to the development of a theory [85].

To avoid the disadvantage mentioned above, according to scholars, gathering a proper sample is more important than having a representative sample. Guo [86] suggested that purposive sampling is about selecting the sample that is the most appropriate for the research objective. Yeh [87] also suggested that researchers could intentionally select a sample that may not represent the population based on their personal experiences. Chu [88] indicated that purposive sampling is about selecting a model sample based on subjective judgments. It is not necessary to use the target population as the population for sampling. Thus, there is no way to calculate sampling errors based on these principles [88]. In spite of this drawback, purposive sampling has been adopted for many national surveys that have yielded meaningful results. Niu [89] explained how purposive sampling should not be limited by research tools; should show openness and flexibility for full, comprehensive, and deep understanding of the research question; and should focus on not only results but also the process of 
how the research question was formed, which helps in summarizing viewpoints and theories based on the research findings.

The main difference between purposive sampling and qualitative sampling is the former's focus on in-depth discussions. Chen [90] suggested that purposive sampling is often used to serve an in-depth research objective, so the results obtained are not as representative as the results of quantitative research. Thus, it is still considered a qualitative research method [90]. Wang [91] further suggested that the purpose of conducting qualitative research is to learn more about the research topic instead of obtaining a general result. This description captures the spirit of qualitative research as well as the essence of exploratory research. Wang [91] believed that purposive sampling could be applied in two types of situations: (a) When conducting a study with deep understanding of the research topic where it is not possible to confirm the boundaries of the population [92] and (b) when the sampling must be confirmed by the researcher due to specific research requirements in order to reflect a certain population while the findings are used for specific inferences and objectives [93]. The difference between these two situations is that in the former sampling tends to be rather passive while in the latter it tends to be rather active. The similarity is that in both types of situations, researchers already have a deep understanding of their research topics.

Two scholars have provided more thoughts on the practice of purposive sampling. Feng [94] noted that purposing sampling is completed based on a researcher's subjective norms and judgments. The sample obtained is a model sample. According to Feng [94], this sampling method is a non-random sampling method. Therefore, purposive sampling can also be called planned sampling. Since the sampling is based on a plan, it is not possible to control errors using probabilities [93]. Another scholar had a similar explanation regarding model samples. Liu [95] believed that purposive sampling is centered on researchers selecting a group of the most representative people for survey based on their logic, common sense, and subjective judgments as well as the subjects' values [95].

According to the 30 scholars' viewpoints regarding the academic research methods described above, it is obvious that purposive sampling, a type of qualitative sampling method, should be applied in this study, which is an exploratory work. This method can be applied to explore the applications of Zhuangzi's concept of self-adaptation to mental health issues. Self-adaptation and mental health are inseparable and often coexist. This research question has two main facets: The initial exploration of the topic and an attempt to develop applications in educational theories. Moreover, this question addresses social development by exploring the range of teaching materials, including literature on history and cultures, in order to offer solutions for practical issues in the field of education.

According to the above, based on many scholars' viewpoints, it is clear that purposive sampling is suitable for the second phase of this study. This study selected a sample of respondents that would not lead to serious errors for the online questionnaire. Sufficient information on the topic was provided to the respondents, and they willingly participated in the survey. If any of the respondents strongly refused to participate or could not take the survey seriously, they chose not to be involved at all or left in the middle of the survey. Thus, this study followed the principles of purposive sampling instead of choosing a representative sample as qualitative studies often do. As a result, reliability analysis was not necessary [76]. Moreover, research ethics were taken into consideration. The respondents were fully informed of the research objective, and their free will was respected with regard to participation. They could choose not to participate any time they wanted. Huang [96] suggested that qualitative research could consider both the deep perceptions of the respondents and the entirety of the findings, covering qualitative and quantitative phenomena and meanings through analyses of the elements involved, the relationships amongst the elements, and the functions of the research, including the research purpose. Thus, the results from the second phase of this study were presented using 3D pie charts with percentage information to display the percentage ratios of the survey results in their entirety and broken down into individual elements. This was done to offer clear visualizations for educators to use as references. 
In the second section of the study, the aim was to collect university students' opinions on the qualitative research. Thus, the results from the first section, which comprised the content categorized based on its relation to the idea of self-adaptation in the Zhuangzi, were used as the foundation for an online questionnaire. Students could access the questionnaire using their smartphones, and they participated in filling out the questionnaire on a voluntary basis. The data collected were then compiled. They were presented in the research findings in the form of percentages based on the categories summarized in the first section. In order to identify whether the concept of self-adaptation from the Zhuangzi was helpful to student learning and for their future development, three extra items were included at the end of the questionnaire. There were two multiple-choice questions, "Which of the self-adaptation categories from the Zhuangzi is the most helpful for mental health?" and "Is it beneficial for modern people's mental health?", and one multiple-answer question, "In which kinds of frustrating situations can self-adaptation be helpful?". For the purpose of designing the online questionnaire, this study collected information about actual cases of hardship in contemporary society, selected tragedies caused by factors unrelated to mental health, and summarized this information to define the categories for the survey. These categories were the options survey participants could select to indicate when self-adaptation was helpful in a similar situation.

The online questionnaire targeted students from Chang Jung Christian University. The goal for student participation in the questionnaire was to recruit at least 350 students from at least three colleges and nine departments as respondents. The students used their own smartphones to fill out the questionnaire. At least one option for each item had to be selected for statistical analysis. Thus, one of the limitations of this study is that "no comment" and "none of the above" were not included as options. The students were asked to truly think of an answer since completing a questionnaire in the field of philosophy requires reflection. If a student could not find a satisfying option, there was always the choice to quit the survey and not submit any responses. Student participation was voluntary. To make the subsequent pie charts easier to read, the order number for each item in a category was included on each corresponding pie chart slice.

\section{Results}

The research findings were obtained using either qualitative or quantitative research methods, as previously discussed. Section 3.1 presents the results of the qualitative research. We used four tables to show the findings for the first four research questions. Coding was used for each chapter to accurately represent the results. For example, the letter A was used for the seven inner chapters (Chapters 1 to 7 in the Zhuangzi), so A1 stands for Chapter 1 of the inner chapters, A2 for Chapter 2 of the inner chapters, and so on. Meanwhile, the letter B was used for the 15 outer chapters (renumbered Chapters 1 to 15, originally Chapters 8 to 22 in the Zhuangzi), so B1 stands for Chapter 1 of the outer chapters, B2 for Chapter 2 of the outer chapters, and so on. Finally, the letter $C$ was used for the 15 miscellaneous chapters (renumbered Chapters 1 to 11, originally Chapters 23 to 33 in the Zhuangzi), so $\mathrm{C} 1$ stands for Chapter 1 of the miscellaneous chapters, C2 for Chapter 2 of the miscellaneous chapters, and so on. The findings concerning concepts related to self-adaptation were as follows in Sections 3.1.5.1 to 3.1.5.11. Section 3.2 presents the results of the quantitative research. The findings of the online questionnaire were based on 397 university students' responses. The survey asked students about the concept of self-adaptation found in the Zhuangzi according to the 11 categories found in Sections 3.1.5.1 to 3.1.5.11. The results pertaining to what was most beneficial for their mental health can be found in Sections 3.2.1-3.2.11. These quantitative research findings answer the fifth research question. The survey results were presented using graphs. The online questionnaire was open from November 2018 to January 2019. During this period of more than two months, a total of 397 university students filled out the questionnaire and submitted their responses. 


\subsection{Results of the Qualitative Research}

\subsubsection{The Frequency of Self-Adaptation Concepts in the Inner Chapters}

There are 31 self-adaptation concepts in the seven inner chapters; the number of times these concepts appeared in each chapter is shown in Table 1.

Table 1. The frequency of self-adaptation concepts in the inner chapters.

\begin{tabular}{cccccccc}
\hline Code & A1 & A2 & A3 & A4 & A5 & A6 & A7 \\
\hline Frequency & 5 & 5 & 3 & 7 & 4 & 6 & 1 \\
\hline
\end{tabular}

Of the seven inner chapters, "In the Human World" (A4) has the highest frequency of self-adaptation concepts, with relevant information appearing seven times. After that, the number of occurrences in the other inner chapters is listed from highest to lowest as follows: Six in "Great Master" (A6), five each in "Carefree Wandering" (A1) and "On the Equality of Things" (A2), four in "The Way of Nurturing Life" (A3), and just one in "Responding to Kings" (A7).

\subsubsection{The Frequency of Self-Adaptation Concepts in the Outer Chapters}

There are 22 self-adaptation concepts found in the 15 outer chapters; the number of times these concepts appeared in each chapter is shown in Table 2.

Table 2. The frequency of self-adaptation concepts in the outer chapters.

\begin{tabular}{ccccccccc}
\hline Code & B1 & B2 & B3 & B4 & B5 & B6 & B7 & B8 \\
\hline Frequency & 1 & - & 1 & 1 & 2 & 1 & - & 1 \\
\hline Code & B9 & B10 & B11 & B12 & B13 & B14 & B15 & - \\
\hline Frequency & 1 & 3 & - & 3 & 6 & - & 2 & - \\
\hline
\end{tabular}

In the 15 outer chapters, the highest number of occurrences, six in total, appeared in "Mountain Tree" (B13). The number of occurrences for self-adaptation concepts in the other outer chapters is listed from highest to lowest as follows: Three each in "Floods of Autumn" (B10) and "Comfortable Life" (B12), two each in "Heaven and Earth" (B5) and "Travel to the North" (B15), and just one each in "Toes Connected" (B1), "Open the Box to Steal Things" (B3), "Noninterference" (B4), "The Principle of Heaven" (B6), "Strengthening the Will" (B8), and "Mending the Nature" (B9).

\subsubsection{The Frequency of Self-Adaptation Concepts in the Miscellaneous Chapters}

There are 17 self-adaptation concepts in the 11 miscellaneous chapters; the number of times these concepts appeared in each chapter is shown in Table 3.

Table 3. The frequency of self-adaptation concepts in the miscellaneous chapters.

\begin{tabular}{ccccccc}
\hline Code & C1 & C2 & C3 & C4 & C5 & C6 \\
\hline Frequency & 5 & 2 & 1 & 3 & 2 & - \\
\hline Code & C7 & C8 & C9 & C10 & C11 & - \\
\hline Frequency & 1 & 1 & 1 & 1 & - & - \\
\hline
\end{tabular}

Of the 11 miscellaneous chapters, "Geng Sang Chu" (C1) had the highest number of self-adaptation concepts at five occurrences. The number of occurrences in the other miscellaneous chapters is listed from highest to lowest as follows: Three in "External Things" (C4), two each in "Syu Wugwei" (C2) 
and "Implied Story" (C5), and just one each in "Dze Yang" (C3), "Robber Jr" (C7), "Discussion on Swords" (C8), "The Old Fisherman" (C9), and "Lye Yukou" (C10).

\subsubsection{Categorizing the Self-Adaptation Concepts}

Throughout the entire Zhuangzi, 70 self-adaptation concepts were found and grouped into 11 categories: The concept of Tao, nature, understanding the truth, mental state, spirit, morality, attitude, way of life, interpersonal relationships, encounters, and life and death. Each category was represented by a number of sentences from the Zhuangzi, and their distribution across the categories is shown in Table 4.

Table 4. The 11 categories of the self-adaptation concepts.

\begin{tabular}{cccc}
\hline Category & Sentences & Category & Sentences \\
\hline 1. The concept of Tao & 7 & 7. Attitude & 8 \\
2. Nature & 6 & 8. Way of life & 7 \\
3. Understanding the truth & 7 & 9. Interpersonal relationships & 8 \\
4. Mental state & 4 & 10. Encounters & 7 \\
5. Spirit & 7 & 11. Life and death & 5 \\
6. Morality & 4 & - & - \\
\hline
\end{tabular}

Across the 11 categories, there were eight sentences each for the self-adaptation concepts of attitude and interpersonal relationships. Regarding the frequency of relevant sentences for the other categories, they were listed from highest to lowest as follows: Seven sentences each for the concept of Tao, understanding the truth, spirit, way of life, and encounters; six sentences each for nature, five sentences for life and death, and four sentences each for mental state and morality.

\subsubsection{The Categorization}

According to our findings, 70 concepts related to self-adaptation were found in the Zhuangzi and classified into 11 categories (Cat. 3.1.5.1-3.1.5.11). Each category contains several relevant sentences. Using concept analysis, these sentences have been organized to present comprehensive and meaningful content, with the chapter reference for each sentence or string of sentences enclosed in parentheses at the end of statements as appropriate. Thus, the results pertinent to the fourth research question are presented as follows.

\subsubsection{The Concept of Tao}

Tao is everywhere (B15_\#1). Tao gathers at the place of modesty and thorough understanding. The so-called mind fast is to remove prejudices, to cleanse and empty the mind (A4_\#2). Fortune gathers in the empty and static mind. The senses of the ears and eyes reach inwards, driving away scheming and calculating (A4_\#3). What is in the future should not be expected, and what is in the past should not be recovered (A4_\#4). If one can be content with the occasion one is in and adapt to changes in one's surroundings, emotions such as sadness and happiness will not enter the mind (A3_\#5). Those who know Tao can reach a noble state without deliberately pursuing loftiness and can cultivate their moral character without deliberately pursuing benevolence and righteousness (B8_\#6). One should follow the laws of nature (A3_\#7).

\subsubsection{Nature}

Sincerity in humanity is the manifestation of real nature (C9_\#1). Great wisdom always looks stupid (B3_\#2). Those without a scheming mind ask for nothing. They are like untied boats floating freely (C10_\#3). A person with opportunistic behaviors will have an opportunistic mind. With an opportunistic mind, one cannot keep a peaceful nature, and therefore one will not be able to follow the rules of Tao (B5_\#4). Nothing can compete with a person of innocent nature in terms of beauty (B6_\#5). 
Ancient true men did not dream while sleeping and did not worry when awake. They did not ask for appetizing food or drink. They breathed deeply (A6_\#6).

\subsubsection{Understanding the Truth}

It is not that the world is small but that men only use the space sufficient to stand in (C4_\#1). Supreme men can achieve the state of Tao without self and things; true men have no fame or fortune in their minds, and divine men do not covet reputation or position (A1_\#2). Fame is subsidiary. Fame is the shadow of trueness (A1_\#3). Those who understand the true meaning of life do not pursue things that cannot avail against life. Those who understand the true meaning of fate do not pursue things they cannot control (B12_\#4). Being greedy for immediate benefit may result in forgetting the importance of life (B13_\#5). Those who know reason must be sensible. Those who are sensible must be clear about adapting to change. Those who are clear about adapting to change must not allow external things to harm them (B10_\#6). Only when one understands reason can one be carefree (A6_\#7).

\subsubsection{Mental State}

Valuing external things results in internal clumsiness (B12_\#1). Hatred, desire, partiality, anger, sadness, and greed can disturb one's innermost being. If one can keep one's innermost being even and straight, one will find peace. Inner peace leads to clarity, inner clarity leads to the state of nothingness, and the state of nothingness leads to conformity to nature (C1_\#2). A supreme man's mind is like a mirror since it allows things to come and go without greeting or farewell and cannot be harmed by external things (A7_\#3). A holy man's mind is peaceful. A peaceful mind leads to contented thoughts. Worries and sufferings are nothing when achieving peace and contentedness. Evil cannot attack his body or mind; his nature will remain complete, and his spirit will not be harmed (C8_\#4).

\subsubsection{Spirit}

The body is the sustenance for the spirit and for it to command (A5_\#1). Human bodies can indeed be like withered branches. Can their minds be like the ashes of a dying fire? (A2_\#2) Heaven and earth exist with me, and all things on earth are one with me (A2_\#3). There are physical changes but no psychological damage (A6_\#8). A person's beauty cannot be seen if she thinks she is beautiful. A person's ugliness cannot be seen if she thinks she is ugly (B13_\#5). If one pays attention to maintaining good health and looks down on fame (C5_\#6), external objects cannot harm one (A1_\#7).

\subsubsection{Morality}

The highest morality is not being influenced by emotions such as sadness and happiness, knowing that to face difficulties is inevitable and still be able to deal with things with a peaceful mind (A4_\#1). Accepting one's own fate calmly is something only a moral person can do (A5_\#2). When a person can surpass others in morality, his physical disability will be forgotten. If people cannot forget the physical forms that they are supposed to forget while also forgetting morality, which they are not supposed to forget, then they are forgetful (A5_\#3). A person of true accomplishment and innocent conduct always feels that he is unclean. A person of true morality can always see his own insufficiencies (C5_\#4).

\subsubsection{Attitude}

One should deal with people and things with an attitude of adaptation and true emotion (B13_\#1). Saying one thing and doing another is illegitimate (A1_\#2). Having wit of the highest level is to be honest with one another without using any wit at all (C1_\#3). People always think they are right at first and then think they are wrong later, so they will adjust in time to adapt to changes in the environment (C3_\#4). When advancing, one should not compete to be first, and when retreating, one should not fall behind (B13_\#5). If you know you are stupid, you are not the most stupid person (B5_\#6). It is very 
dangerous to brag about one's own achievements and offend a superior (C2_\#7). Being smart is to stop when you encounter something you do not understand (A2_\#8).

\subsubsection{The Way of Life}

Things in the world should be listened to not with the ears but with the heart (A4_\#1). There are times of difficulty for those with quick wit (C4_\#2). Moral principles should be followed by everyone (A4_\#3). Those with broad knowledge are open-minded and never argue with others, while those with limited knowledge always haggle over every ounce (A2_\#4). Give up small knowledge, and profound knowledge will appear. One should get rid of the firm belief that one is kind, and then kindness will manifest (C4_\#5). If a person can fulfill his own duty and know what he is not capable of, he is sensible and wise (C1_\#6). The purpose of doing virtuous deeds recognized by the world is not to gain reputation, and the purpose of doing bad deeds recognized by the world is not to avoid punishment. The normal practice is to go with the law of nature. It helps to protect lives and nature and keeps good health for longevity (A3_\#7).

\subsubsection{Interpersonal Relationships}

Nobody will want to get close to a person who cannot tolerate others. Those who nobody wants to get close to will be left alone (C1_\#1). One must achieve the state of not feeling excited when being praised by the entire world and not feeling depressed when being criticized by the entire world (A1_\#2). If you push someone too hard, he may demand vengeance (A4_\#3). The harm caused by weapons is not as serious as that caused by me (C1_\#4). If you just follow others blindly, you will never experience real joy (B1_\#5). One should not show one's nobility to loathe others or flaunt one's wealth to tease others (C7_\#6). People who get together because of profit will leave each other in a lurch. People who get together because of their natures will take care of each other. Taking care of each other is far from leaving each other (B13_\#7). A gentleman's friendship is as insipid as water. A villain's friendship is as delectable as sweet wine. A gentleman's affection is insipid but long and intimate. A villain's affection is delectable but fragile (B13_\#8).

\subsubsection{Encounters}

The static state is the optimal state of water when it stops. It can be an example to follow. Keeping calm inside can avoid the turbulence caused by external interference (A5_\#1). When dominated by emotions such as joy and anger and by one-sided views, different perspectives may be generated (A2_\#2). To truly rule external things, one must dominate them without being enslaved by them (B4_\#3). We should not be influenced by the environment and should change our ideas into peaceful ones (B12_\#4). We should not indulge ourselves for exalted positions and great wealth and should not play up to those in power because of poverty (B9_\#5). We should be calm inside when facing something disastrous or cheerful (B10_\#6). A holy man's bravery is to know that poverty is caused by fate and that greatness is caused by opportunity (B10_\#7).

\subsubsection{Life and Death}

All things rise and flourish. They grow. All things change and decline. They die (B15_\#1). Nature gives me my physical body, makes me work for a living, allows me to be easy and happy in my later years, and grants me peace when I die. Birth and death are like dreams. Dying is like easing into sleep. Being born is like waking up with a start from a dream (A6_\#2). Those who can treat life kindly can die peacefully (A6_\#3). Life and death are a cycle. Life and death come and go. All men can do is to allow them to take their natural course (A6_\#4). Things will develop in the opposite direction when they become extreme. An end is also a beginning. This is the common rule for all things (C2_\#5). 


\subsection{Results of the Purposive Sampling}

In total, 397 students participated in the online questionnaire. The respondents represented five colleges/schools: The College of Continuing Education, the College of Health Sciences, the College of Humanities and Social Sciences, the College of Information and Design, and the College of Management. Moreover, the participants came from the following fourteen departments: The Bachelor's Degree Program in Environment and Food Safety Laboratory Science; the Bachelor's Degree Program in Fire Safety Science; the Bachelor's Degree Program in Japanese; the Bachelor's Degree Program in Management; the Department of Applied Japanese Language; the Department of Aviation and Maritime Transportation Management; the Department of Computer Science and Information Engineering; the Department of Finance; the Department of Fine Arts; the Department of Mass Communication; the Department of Nutrition and Health Sciences; the Department of Tourism, Food, and Beverage Management; the Department of Translation and Interpretation Studies; and the Medical Healthcare Industry Bachelor's Degree Program.

\subsubsection{Content for the Concept of Tao}

This category contained seven items. Most of the students (79 students) found the sixth item to be the most helpful. The other items are listed from highest to lowest as follows: 71 students found the fifth item to be the most helpful, 56 found the seventh item to be the most helpful, 53 found the second item to be the most helpful, 53 found the third item to be the most helpful, and 44 found the first item to be the most helpful. In comparison, the fewest number of students ( 41 students) found the fourth item to be the most helpful. The relevant percentages are shown in Figure 1.

\section{Cat. 3.1.5.1}

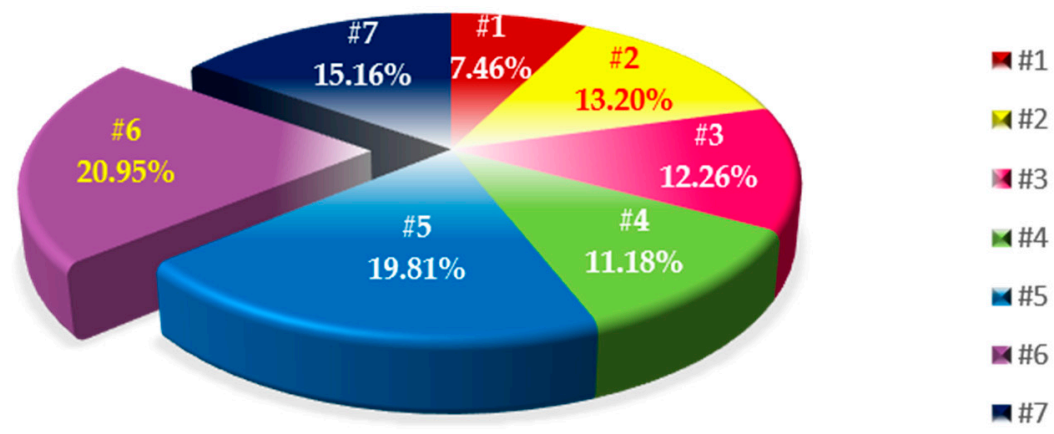

Figure 1. The distribution of students who thought one of the seven items related to the concept of "Tao" was the most helpful.

\subsubsection{Content for Nature}

This category contained six items. Most of the students (131 students) found the fourth item to be the most helpful. The other items are listed from highest to lowest as follows: 83 students found the fifth item to be the most helpful, 75 found the first item to be the most helpful, 49 found the fourth item to be the most helpful, and 35 found the sixth item to be the most helpful. In comparison, the fewest number of students ( 24 students) found the second item to be the most helpful. The relevant percentages are shown in Figure 2. 


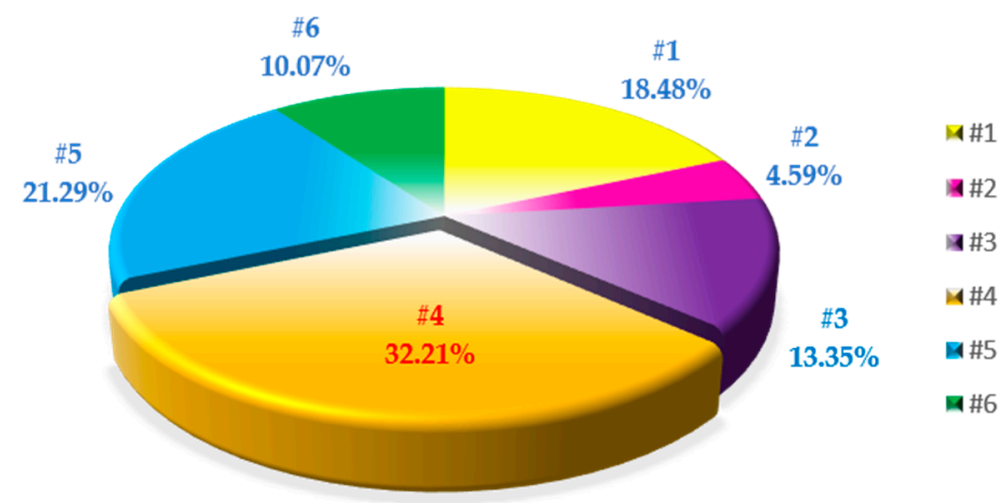

Figure 2. The distribution of students who thought one of the six items related to nature was the most helpful.

\subsubsection{Content for Understanding the Truth}

This category contained seven items. Most of the students (136 students) found the fourth item to be the most helpful. The other items are listed from highest to lowest as follows: 113 students found the sixth item to be the most helpful, 45 found the second item to be the most helpful, 42 found the first item to be the most helpful, 32 found the seventh item to be the most helpful, and 16 found the fifth item to be the most helpful. In comparison, the fewest number of students (13 students) found the third item to be the most helpful. The relevant percentages are shown in Figure 3.

\section{Cat. 3.1.5.3}

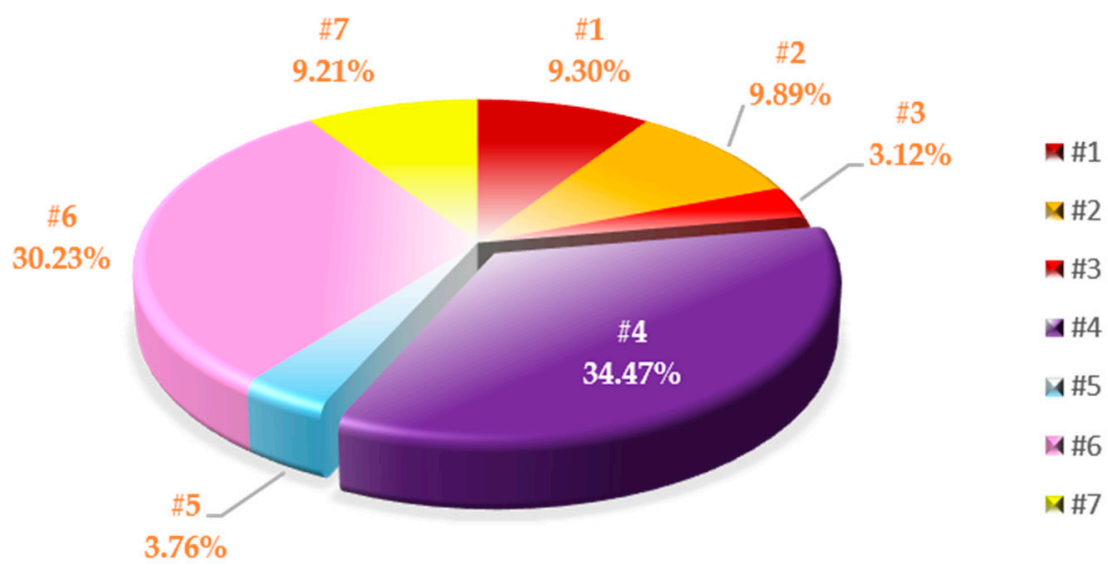

Figure 3. The distribution of students who thought one of the seven items related to understanding the truth was the most helpful.

\subsubsection{Content for Mental State}

This category contained four items. Most of the students (211 students) found the second item to be the most helpful. The other items are listed from highest to lowest as follows: 93 students found the fourth item to be the most helpful and 57 found the third item to be the most helpful. In comparison, the fewest number of students (36 students) found the first item to be the most helpful. The relevant percentages are shown in Figure 4. 


\section{Cat. 3.1.5.4}

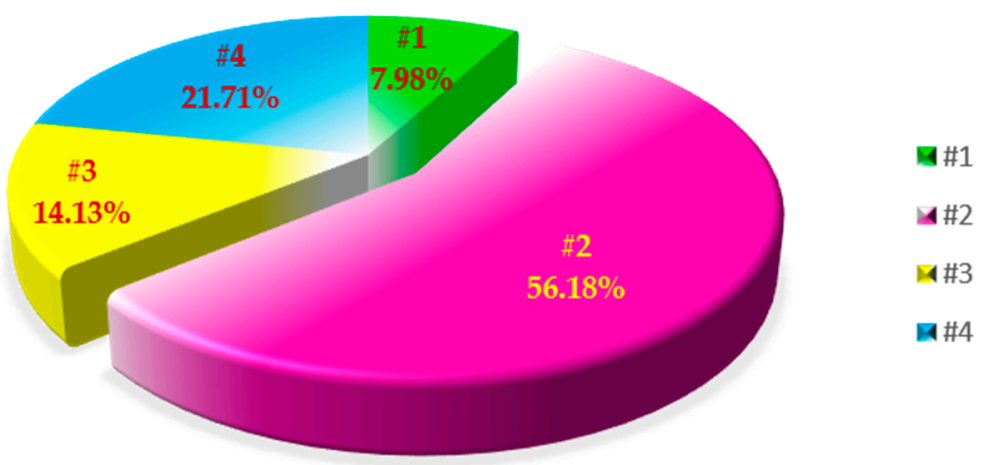

Figure 4. The distribution of students who thought one of the eight items related to mental state was the most helpful.

\subsubsection{Content for the Spirit}

This category contained seven items. Most of the students (142 students) found the fourth item to be the most helpful. The other items are listed from highest to lowest as follows: 97 students found the third item to be the most helpful, 66 found the second item to be the most helpful, 41 found the first item to be the most helpful, 20 found the fifth item to be the most helpful, and 17 found the sixth item to be the most helpful. In comparison, the fewest number of students (14 students) found the seventh item to be the most helpful. The relevant percentages are shown in Figure 5.

\section{Cat. 3.1.5.5}

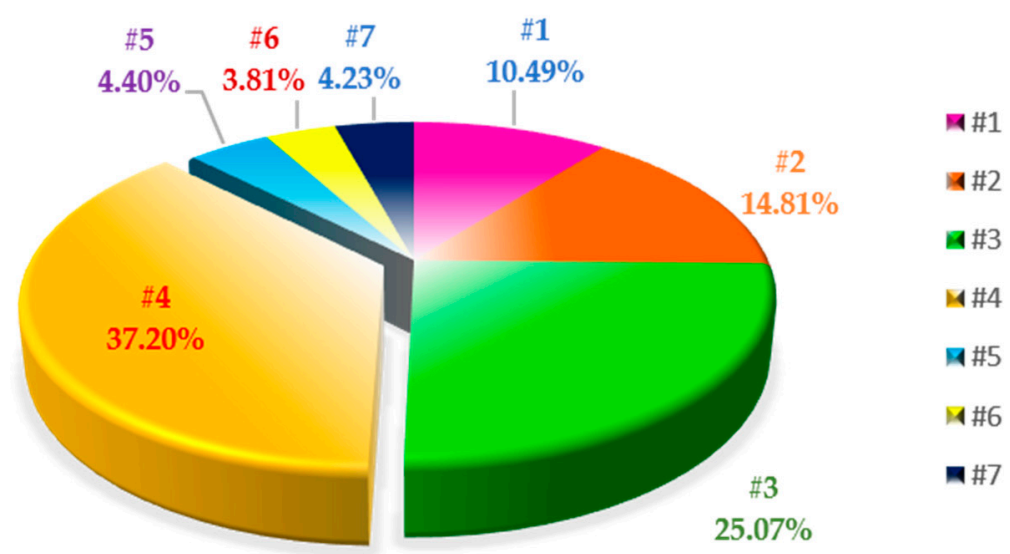

Figure 5. The distribution of students who thought one of the seven items related to the spirit was the most helpful.

\subsubsection{Content for Morality}

This category contained four items. Most of the students (128 students) found the first item to be the most helpful. The other items are listed from highest to lowest as follows: 112 students found the fourth item to be the most helpful and 83 found the third item to be the most helpful. In comparison, the fewest number of students (72 students) found the second item to be the most helpful. The relevant percentages are shown in Figure 6. 


\section{Cat. 3.1.5.6}

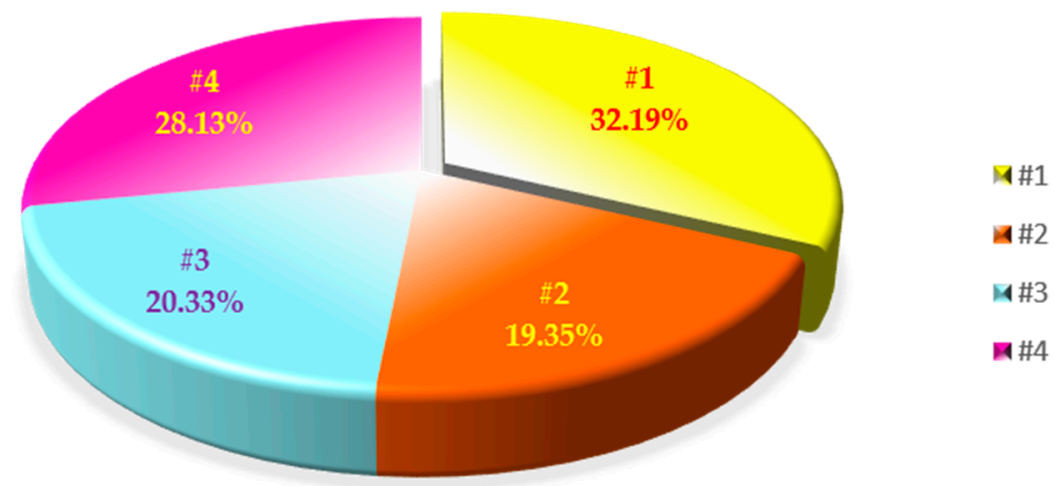

Figure 6. The distribution of students who thought one of the four items related to morality was the most helpful.

\subsubsection{Content for Attitude}

This category contained eight items. Most of the students (164 students) found the first item to be the most helpful. The other items are listed from highest to lowest as follows: 92 students found the fourth item to be the most helpful, 64 found the eighth item to be the most helpful, 24 found the third item to be the most helpful, 24 found the third item to be the most helpful, 22 found the sixth item to be the most helpful, 14 found the second item to be the most helpful, and 14 found the fifth item to be the most helpful. In comparison, the fewest number of students (three students) found the seventh item to be the most helpful. The relevant percentages are shown in Figure 7.

\section{Cat. 3.1.5.7}

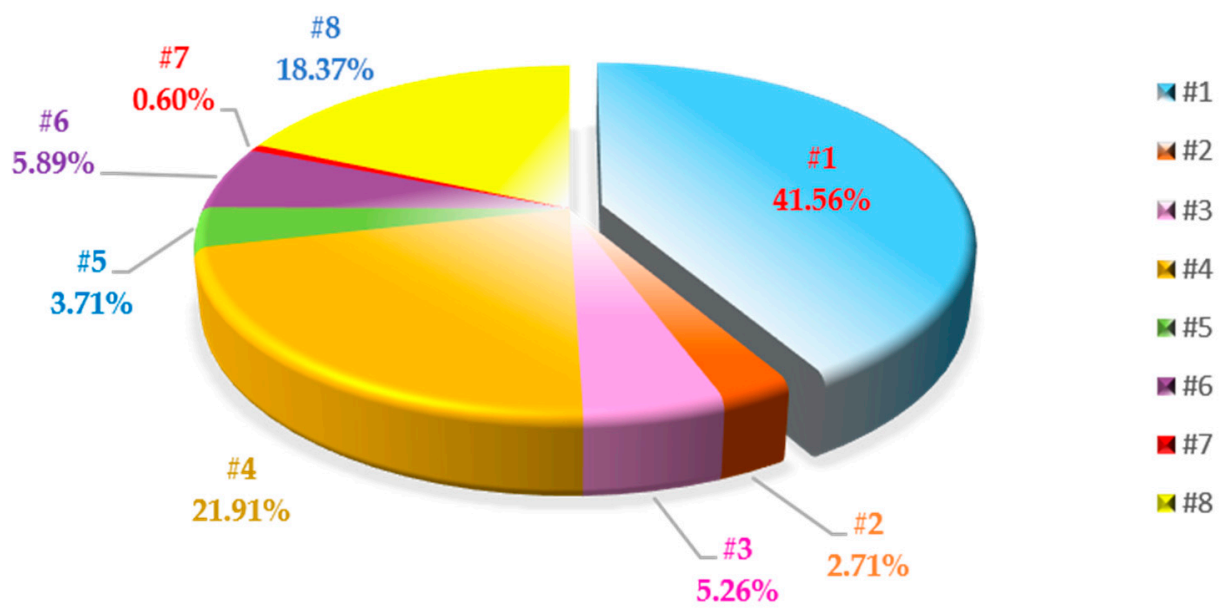

Figure 7. The distribution of students who thought one of the eight items related to attitude was the most helpful.

\subsubsection{Content for the Way of Life}

This category contained seven items. Most of the students (114 students) found the first item to be the most helpful. The other items are listed from highest to lowest as follows: 106 students found the seventh item to be the most helpful, 66 found the fourth item to be the most helpful, 53 found the sixth item to be the most helpful, 23 found the fifth item to be the most helpful, and 20 found the third item to be the most helpful. In comparison, the fewest number of students (15 students) found the second item to be the most helpful. The relevant percentages are shown in Figure 8. 


\section{Cat. 3.1.5.8}

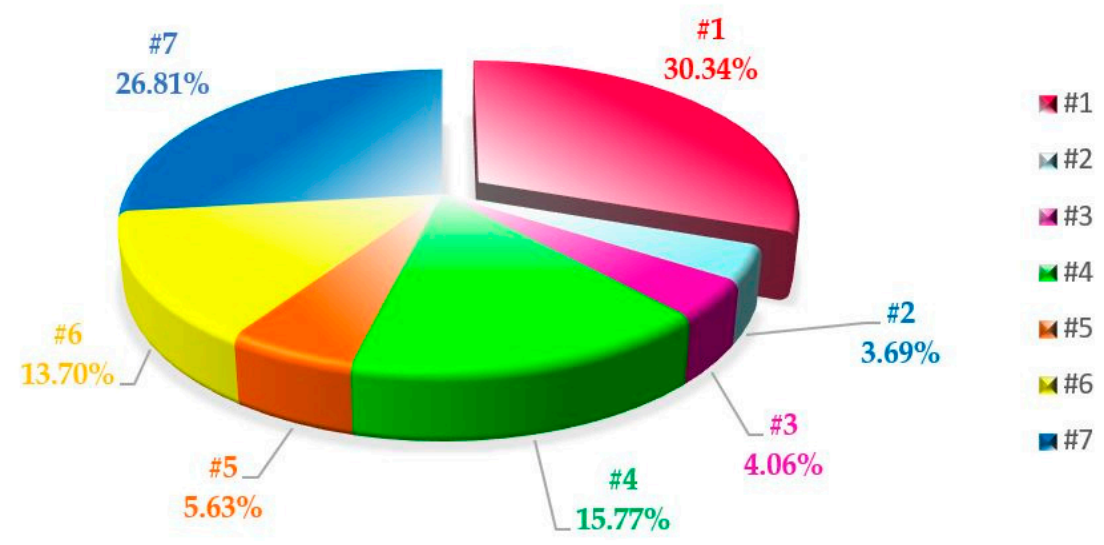

Figure 8. The distribution of students who thought one of the eight items related to the way of life was the most helpful.

\subsubsection{Content for Interpersonal Relationships}

This category contained eight items. Most of the students (95 students) found the eighth item to be the most helpful. The other items are listed from highest to lowest as follows: 64 students found the sixth item to be the most helpful, 61 found the second item to be the most helpful, 57 found the fifth item to be the most helpful, 51 found the seventh item to be the most helpful, 29 found the first item to be the most helpful, and 21 found the fourth item to be the most helpful. In comparison, the fewest number of students (19 students) found the third item to be the most helpful. The relevant percentages are shown in Figure 9.

Cat. 3.1.5.9

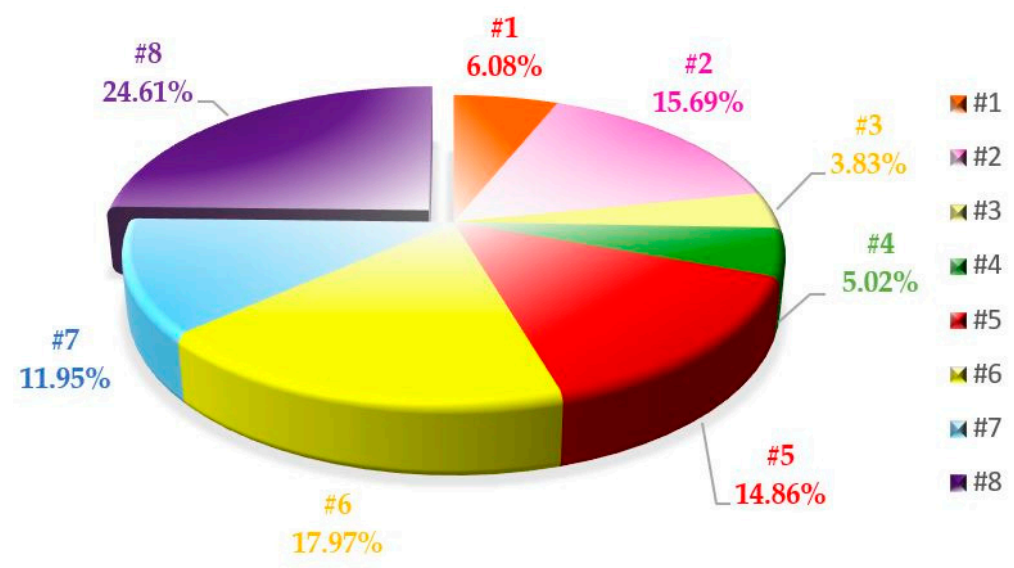

Figure 9. The distribution of students who thought one of the eight items related to interpersonal relationships was the most helpful.

\subsubsection{Content for Encounters}

This category contained seven items. Most of the students (110 students) found the first item to be the most helpful. The other items are listed from highest to lowest as follows: 96 students found the fourth item to be the most helpful, 59 found the seventh item to be the most helpful, 54 found the fifth item to be the most helpful, 37 found the second item to be the most helpful, and 26 found the third item to be the most helpful. In comparison, the fewest number of students (15 students) found the sixth item to be the most helpful. The relevant percentages are shown in Figure 10. 


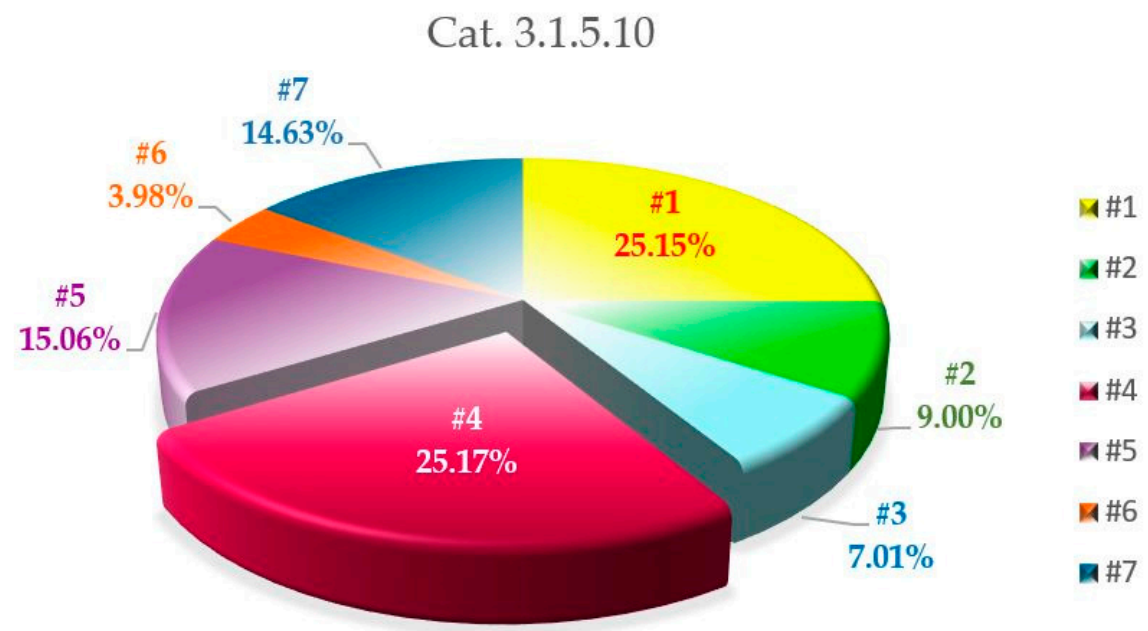

Figure 10. The distribution of students who thought one of the seven items related to encounters was the most helpful.

\subsubsection{Content for Life and Death}

This category contained five items. Most of the students (117 students) found the first item to be the most helpful. The other items are listed from highest to lowest as follows: 109 students found the second item to be the most helpful, 64 found the fifth item to be the most helpful, and 55 found the fourth item to be the most helpful. In comparison, the fewest number of students ( 52 students) found the third item to be the most helpful. The relevant percentages are shown in Figure 11.

\section{Cat. 3.1.5.11}

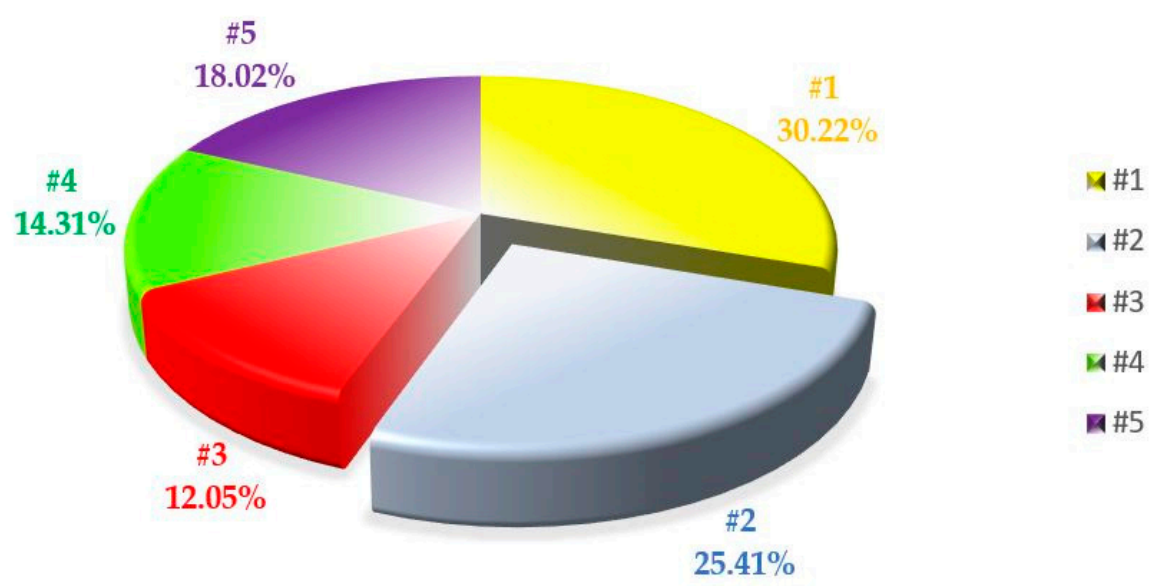

Figure 11. The distribution of students who thought one of the five items related to life and death was the most helpful.

\section{Discussion}

The research results presented the concepts of self-adaptation found in the Zhuangzi after they were categorized into 11 different topics. Although the different categories presented ideas pertaining to a specific topic, the core value supporting each category was related to self-adaptation. In other words, although there were 11 separate topics, the meanings of the topics were interconnected and not independent of one another. In the Zhuangzi, the idea of self-adaptation is central to the realization of the philosophy of life, and the philosophy underlying self-adaptation is always inspired by nature. Indeed, this inspiration drawn from nature pertains to the wisdom of peace in both its physical and psychological aspects, and wisdom is what this study aims to explore with respect to self-adaptation. 
Even though parts of the research results were abstract—-they need exploring and discussing —all the content was interpreted according to Zhuangzi's ideas so that readers could better understand the theory of self-adaptation.

Since Zhuangzi's philosophy is a philosophy of life, the logical follow-up question is, how can one learn to self-adapt? The first step is to remove any restrictions on the mind. Stains on the mind must be erased. What are these stains? Zhuangzi believed that one kind of stain is preference. He said that we should not harm ourselves because of our preferences. Feeling pleasantly surprised or resentfully angry will harm one's nature. For example, in the chapter "In the Human World", there is a story about a strange person named Jr Lishu. His chin was lower than his navel, and his shoulders were located above his head. His bun pointed to the sky, and the acupuncture points of his internal organs and blood vessels were enlarged. However, Jr Lishu could still provide for himself and lived a long life. Zhuangzi praised Jr Lishu's spirit for not giving up. If you have a natural life, what is there to abandon? The ugly man did not know his ugliness and forgot what he looked like; he forgot, too, that people would laugh at him because of his physical features. All he knew was that he enjoyed his natural life. Zhuangzi believed that living in this way was complete morality, for it is beyond appearance and preference. It is the true essence of self-adaptation.

Any stains created by the mind can only be erased by the mind. This is the meaning of the mind fast. According to the Zhuangzi, humankind's emotional desires cannot be satisfied. Thus, it is impossible to have inner peace when a person is filled with desire. If he cannot have inner peace, he cannot show his true nature. Thus, the Zhuangzi tells us to cultivate our minds-in other words, to practice mind fasting. If we can erase the stains caused by humankind's world and preserve nature, we will not fall into the paradoxical traps in the human world and will be rid of all worries. For example, people should check their reflections in still water instead of running water. A peaceful mind is like still water because it shows one's true self. According to the Zhuangzi, supreme individuals never seek fame or wealth. They are peaceful inside like a clear mirror. What they do is reflect external things naturally while not being influenced by them. This is the principle that Zhuangzi stressed: Keeping good health and reconciling oneself to one's situation.

The fables and stories in the Zhuangzi are still relevant to our daily lives. The material Zhuangzi used for literal descriptions contains inspirational principles of life. In other words, Zhuangzi wrote fables based on the various difficulties he faced in his own life, and he integrated philosophy, literature, and art into his writing. He used critical writing by comparing different situations and contrasting them and including ironic allusions to humanity. Zhuangzi was skilled in creating fables that embraced stark contrasts regarding different forms and capacities so that readers could easily see how conflicts were caused by differences. For example, on one occasion in his life, Zhuangzi asked an official for a loan since he had no money to buy food. The official said that he would lend Zhuangzi a considerable sum after collecting taxes from the people, and Zhuangzi replied by telling him the following story. Once, he saw a fish that was about to die because there was no water. The fish asked a man for a sip of water to survive. The man told the fish that it would be able to swim freely after the man had channeled water from the river. The fish said that all it needed was a small sip of water and that without the sip it would die. Then Zhuangzi explained to the official that his offer was no better than the offer the man had made to the fish. This story illustrates the significance of Zhuangzi's original request for a small amount of food at the immediate moment being met with an offer of a huge amount of money later. Zhuangzi's biting humor highlights the hypocrisy of humanity: In this case, the sip of water symbolizes life while abundant water from the river symbolizes death. This is an illustrative example of Zhuangzi's skill in using a simple story to make a vivid point through the combination of art, irony, and philosophy.

\section{Conclusions}

Zhuangzi was a central thinker in ancient China as well as a representative figure of the Taoist schools. He had a deep understanding of the philosophy of life. Zhuangzi used Laozi's ideas but 
rendered them less abstract by providing both theoretical and practical applications. Zhuangzi's concept of self-adaptation is not simply a logical deduction but contains original insights into how we can transcend adverse circumstances.

This study analyzed the concept of self-adaptation in his work, the Zhuangzi, and found 70 occurrences of relevant concepts: 31 in the seven inner chapters, 22 in the 15 outer chapters, and 17 in the 11 miscellaneous chapters. The top five chapters, according to the number of occurrences, are "In the Human World" with seven occurrences, "Great Master" and "Mountain Tree" with six occurrences each, and "Carefree Wandering" and "On the Equality of Things" with five occurrences each. After concept analysis and summary, the 70 occurrences of self-adaptation concepts were categorized into 11 categories. The content of each category was then ordered in a logical way to create a piece of text that could help guide a reader's general understanding of the category and mastery of the self-adaptation concept.

A similarity between the environment Zhuangzi lived in and modern culture is the fact that people from both eras have been facing trying situations in life and at work. However, in Zhuangzi's time, life was much more difficult. Even so, Zhuangzi was able to maintain a happy mental state, and credit for this should be given to his ability to transcend the worldly through his state of mind. However, this transcendence did not happen overnight. It required passing severe tests, putting forth his best efforts in difficult situations, realizing the wisdom in life, and finally achieving real mental happiness. Zhuangzi obtained this ultimate happiness through the power of self-adaptation, which helped him overcome all forms of depression. This study analyzed the book the Zhuangzi and grouped 70 self-adaptation concepts into 10 categories that were then used to design the online questionnaire for the empirical study. It was expected that sharing the study's findings with the general public would help modern people deal with the challenges they face in life and at work. By improving their self-adaptation skills, people will become better at maintaining happy mental states even in trying situations. This was the study's biggest contribution.

In order to further explore the statistical meanings of the university students' feedback on which pieces of content were the most helpful in frustrating situations, the maximum values, minimum values, means, and standard deviations of the 70 items and 11 categories analyzed are presented in Table 5 .

Table 5. The statistics of the 11 categories.

\begin{tabular}{cccccc}
\hline Category & Items & Max & Min & Mean & SD \\
\hline $\mathbf{1}$ & 7 & 79 & 41 & 56.7143 & 12.7471 \\
$\mathbf{2}$ & 6 & 131 & 24 & 66.1667 & 35.6109 \\
$\mathbf{3}$ & 7 & 136 & 13 & 56.7143 & 44.7013 \\
$\mathbf{4}$ & 4 & 211 & 36 & 99.2500 & 67.6623 \\
$\mathbf{5}$ & 7 & 142 & 14 & 56.7143 & 44.6885 \\
$\mathbf{6}$ & 4 & 128 & 72 & 98.7500 & 22.3313 \\
$\mathbf{7}$ & 8 & 164 & 3 & 49.6250 & 51.4974 \\
$\mathbf{8}$ & 7 & 114 & 15 & 56.7143 & 37.8633 \\
$\mathbf{9}$ & 8 & 95 & 19 & 49.6250 & 24.0673 \\
$\mathbf{1 0}$ & 7 & 110 & 15 & 56.7143 & 32.6615 \\
$\mathbf{1 1}$ & 5 & 117 & 52 & 79.4000 & 27.8324 \\
\hline
\end{tabular}

SD: Standard deviation.

The standard deviations in this table represent the discrete degrees of the 397 students' responses for the items in the 11 categories. The discrete degree of the top three items from the fourth category was the highest, followed by the seventh category. The third category and the fifth category were ranked in third place, with almost equal discrete degrees. They were followed by the eighth category, the second category, the tenth category, and the eleventh category. The students' responses for the ninth category and the sixth category were not very different. Finally, the discrete degree for the first category was the lowest. In other words, the students' responses for this category were very similar. 
The highest discrete degree (for the fourth category) was 5.3 times higher than the degree for the lowest one (for the first category). In sum, the students' views on these categories were quite different. Therefore, when implementing these categories in teaching activities, instructors must keep an open mind in order to accept individual differences and guide their students in finding the learning options that best suit them based on their subjective views.

To exam how beneficial students thought the Zhuangzi content would be in helping them get through frustrating situations in contemporary, three additional questions were included in the survey. The first extra question was, which of the self-adaptation categories from the Zhuangzi is the most helpful for mental health? Of the 11 categories, most of the students (162 students) found that self-adaptation was most helpful in situations from the fourth category. Seventy-two, 28, 27, 22, 20, 17, 15,14 , and 13 students found that self-adaptation was the most helpful in situations from the seventh, eighth, first, sixth, third, fifth, ninth, tenth, and eleventh categories, respectively. In comparison, the fewest number of students (seven students) found that self-adaptation was the most helpful in situations from the second category. The bar graph in Figure 12 presents the relevant results.

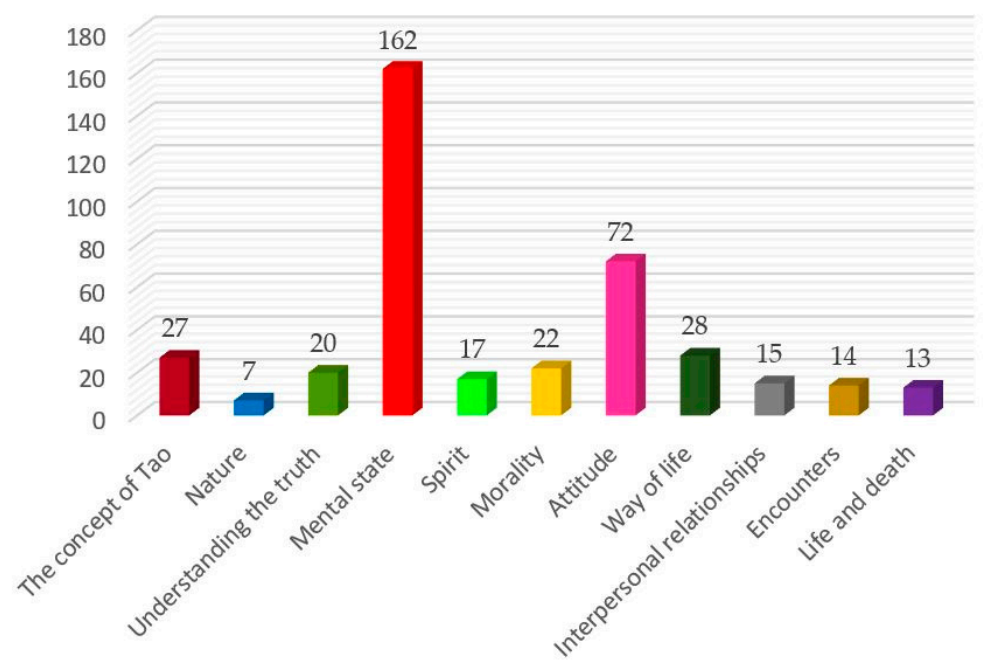

Figure 12. The most helpful self-adaptation category for mental health in frustrating times.

Zhuangzi has received a lot of attention amongst scholars around the world in part because of its focus on regimen. One contribution of this study was the embodiment of a Taoist regimen consisting of 70 items in 11 categories to support positive mental health practices in contemporary life. The students commented on whether they agreed that self-adaptation could be helpful for improving their mental health. According to the results of the online questionnaire, of the 397 university students who responded, 146 students strongly agreed that self-adaptation was helpful for improving mental health. Meanwhile, 188 students agreed, 52 neither agreed nor disagreed, seven disagreed, and four strongly disagreed with the statement that the Taoist concept of self-adaptation was helpful for mental health, meaning that most of the university students found the concept helpful when they faced trying situations. The relevant percentages are shown in Figure 13. 


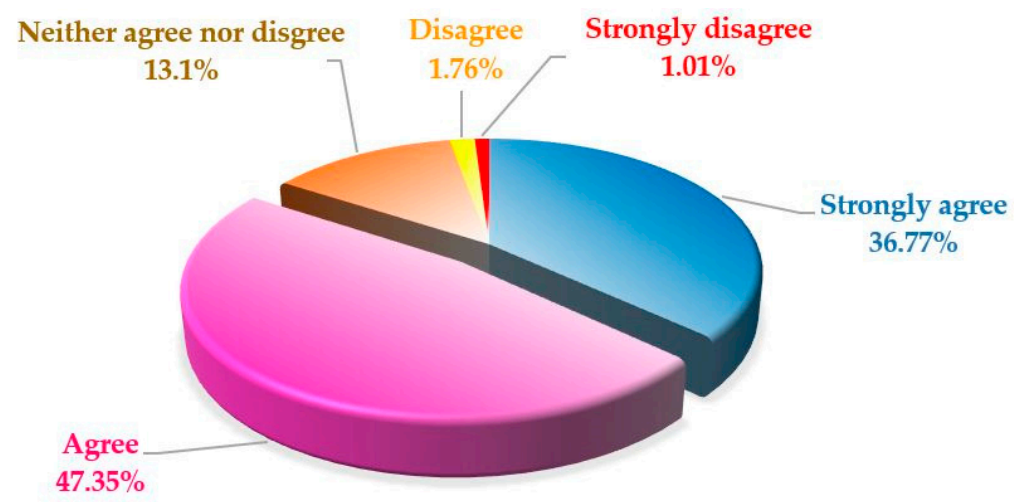

Figure 13. The distribution of student responses regarding whether they agreed that self-adaptation could be helpful for mental health when they faced frustrating situations.

The last question, Item \#14, was a follow-up question. Items \#1 through to \#13 of the online questionnaire were all multiple-choice questions. The only multiple-answer question was Item \#14, with the options being situations designed based on real-life cases of tragedies caused by a factor not related to mental health in contemporary society. These situations were designed to allow the students to think freely about whether self-adaptation would be helpful in similar situations. This study presented a total of eight situations: Workplace situations (\#1), love situations (\#2), family situations (\#3), interpersonal relationship situations (\#4), situations with economic pressure (\#5), situations involving pride (\#6), situations of being bullied (\#7), and accident situations (\#8). These categories for different situations were used as the options for the question, in which kinds of frustrating situations can self-adaptation be helpful? The results of the online survey show that most of the students (353 students) found that self-adaptation was the most helpful in interpersonal relationship situations (the fourth category). After that, 314, 291, 227, 221, 183, and 174 students found that self-adaptation was the most helpful in workplace situations (the first category), situations involving pride (the sixth category), family situations (the third category), love situations (the second category), accident situations (the eighth category), and situations of being bullied (the seventh category), respectively. In comparison, the fewest number of students (136 students) found that self-adaptation was the most helpful in situations with economic pressure (the fifth category). The bar graph in Figure 14 presents the relevant results. Using these same answers, it could also be concluded that 89 students (22.41\%) regarded all eight categories as presenting situations where self-adaptation skills could be helpful.

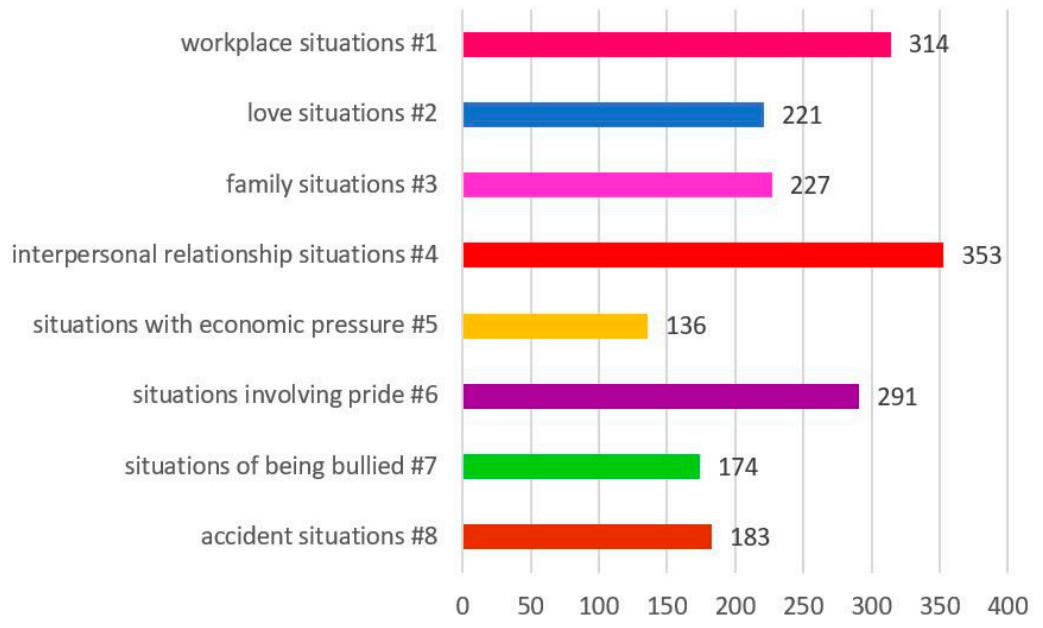

Figure 14. Student feedback on how helpful the concept of self-adaptation would be in eight types of frustrating situations. 
Living means dealing with people, things, events, society, contradictions, crises, and disappointments. In other words, when the external, objective environment collides with our minds, we cannot carry out our own ideas. We often encounter obstructions in reality that are followed by difficulties and worries, and if our worries grow, there is a chance that we will spin cocoons around ourselves and never be free. Thus, according to the Zhuangzi, the act of mental transcendence must be based on an effort to cultivate one's mind. If we can achieve this in the most demanding situations, we can keep peaceful and tranquil minds. A single thought can make a difference by bringing light to a painful situation, which is the essence of transcendence. If one's emotions in a demanding situation can be governed and clarified, the pain disappears, and one has achieved the state of self-adaptation.

Using fables, the Zhuangzi shows the importance of this transcendence, which exists beyond life's framework and continues to grow as a life force. By going beyond the mind and life's finite boundaries, a vulnerable force can turn into a strong one. If the narrow knowledge of a frog in a well can become as broad as the sky, then the value of human life can be redefined and not restricted to our harsh social and cultural environments. Zhuangzi set a goal for us all by encouraging us to pursue the rich, carefree, happy life, which is also the definition of self-adaptation.

Self-adaptation is a kind of autonomous happiness that does not come from outside. Nor can it be given by someone else or provided by the environment. It must grow spontaneously and come from inside. It is not a temporary state of mind but the manifestation of the value of one's core nature. Since Zhuangzi cared about individual gains, losses, advantages, disadvantages, and suffering, he believed that people should learn how to bring peace to their lives based on nature's laws. The origin of nature is Tao, which is why we must learn from nature. According to Zhuangzi, those who practice Tao are morally complete, and the purpose of the Zhuangzi is to remind us that the only way to treat ourselves well is to understand the rules of life and to face every situation positively-thus pursuing continuous self-cultivation. Through this method, people can deal with things morally, understand reasons clearly, face difficulties, preserve even temperaments, and conduct themselves naturally. In other words, when we comprehend morality correctly and our internal cultivation reaches a certain point, we can live a psychological life of self-adaptation.

The value of Zhuangzi's philosophy, especially the concept of self-adaptation, is undefinable. Throughout his life, Zhuangzi suffered, but he learned how to free his mind from sorrow and achieve a peaceful, carefree mental state. The World Health Organization recently reported a dramatic rise in depression [97], which is unsurprising given the challenges of modern life [98,99]. This makes Zhuangzi's philosophy all the more relevant today. Even for the well prepared, life in the 21st century can be challenging, but for many others, it presents insurmountable obstacles and frustrations. When people lose their equanimity, they are influenced by their emotions and unable to deal with life's complexities. How should they approach such problems? Zhuangzi provides an answer: By having an attitude that is guided by self-adaptation. Positive mental health helps us transcend our worldly concerns. This is the principal message of Zhuangzi's philosophy.

This study paid close attention to mental health education and science education, which are two sides of the same coin. In other words, they share a common essence. To be more specific, they are both about identifying real-life problems and trying to solve them. But even though they are the same in nature, some differences remain. A key difference is that science education is about solving problems in the external environment and cultivating abilities related to socialization, while mental health education is about solving psychological problems and ensuring mental health during personal development. Thus, when it comes to education, neither side of the coin should be ignored.

Knowledge, skills, and attitude are the three major concepts mentioned when talking about educational goals. Professionals with knowledge cultivated through education can make positive contributions to society and culture as long as they have good mental health. On the other hand, those with poor mental health often remain in doubt about their capabilities even if they have been well trained. Therefore, modern education should focus on not only knowledge and skills but also helping students to develop positive attitudes towards life. Attitudes depend on mental state. A healthy 
mental state leads to a healthy attitude, and healthy attitudes comprise the cornerstone of a healthy society. They are the foundation for healthy and sustainable cultures.

Modern education tends to focus on cross-field lessons because they help improve student learning results and the ability to solve problems through creative solutions. This study featured not only cross-field teaching and innovative learning but also the use of cross-field teaching materials in a flexible way to extend the range of lesson design to cover all kinds of professional content. Moreover, this study analyzed the Zhuangzi from the perspectives of literature and philosophy and integrated issues related to mental health education into the discussion. The cross-field academic work highlights the importance of taking mental health education into consideration across all disciplines. Figure 15 shows the different contexts for mental health education by summarizing the points previously discussed in the paragraphs above.

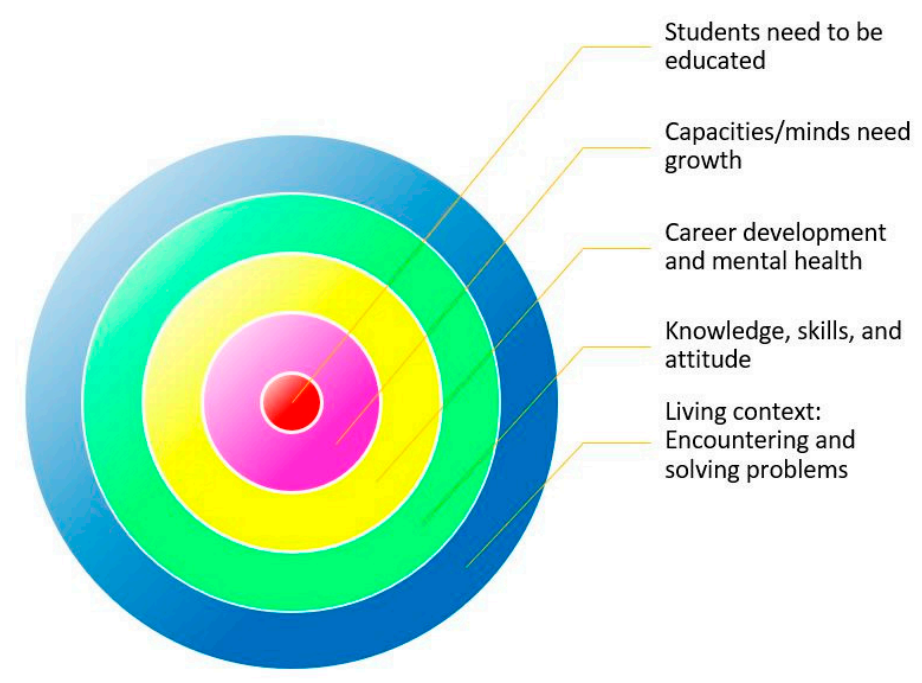

Figure 15. The different contexts for mental health education.

Social phenomena and culture present countless situations where student mental health development may be obstructed. Educators cannot ignore their pupils' mental health. Thus, teachers should not only pay attention to the possible negative influences of the surrounding environment but also consider mental health development by designing teaching materials or courses with mental health activities that students can actually take part in. In other words, educators should pay attention to the factors that may cause a student's negative mindset in daily living contexts since students are likely to feel frustrated in such scenarios. Thus, this study stresses that as educators, teachers should focus on preventive education because students who are feeling despair may also lose their self-confidence. To address this problem, educators should strive to ensure their teaching materials impart life lessons. This study uses Zhuangzi's concept of self-adaptation to enhance student mental health by inspiring positive attitudes and helping students develop their talents. This approach to student success is an educational responsibility for this era and also an educational goal. Figure 16 shows the study's proposed mental health education model.

Of course, few of us can claim to be immune to life's frustrations. Mortality means that we are far from perfect-but neither are we powerless victims, tossed hither and thither on a cruel and indifferent sea. Zhuangzi's philosophy teaches us how to stay positive and maximize our strengths, skills, and abilities. Self-adaptation is a philosophy of doing and being, of character, of overcoming difficulties, and of caring for one's mental and physical health. In contemporary life, Zhuangzi's philosophy has lessons for people of all ages. 


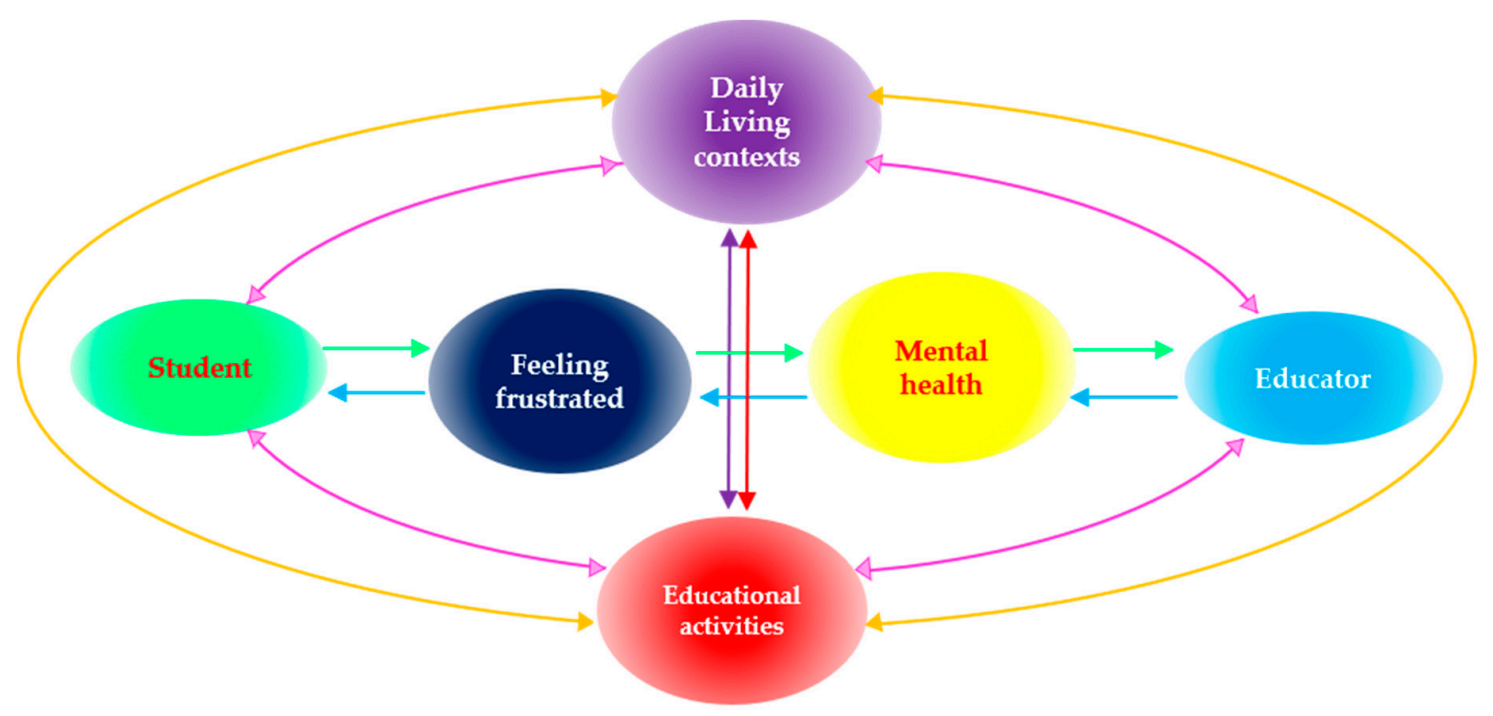

Figure 16. Mental health education model.

Teachers think highly of an education model that puts students at the centre of educational philosophy. The research findings are closely related to student mental health issues, consistent with the student-centered educational approach but with even broader content than typically seen. One way to study student mental health issues is to explore the role of educators and teacher-student interactions through the lens of various fields, such as psychology, sociology, educational psychology, educational sociology, and educational philosophy, to identify ways to improve student mental health both in educational scenarios and in the process of education work. However, the focus should also be on preventing obstacles that might negatively influence students by identifying potential obstructions and addressing them before they become severe to the degree that students cannot achieve their full potential or make full use of their talents. This approach reflects the new educational issues that have arisen as result of modern society. Educators and those who work in education in any capacity should not ignore the issues borne from our high-pressure society.

It is undeniable that educators play a role in passing on culture and innovating the learning process. They shoulder the responsibility of developing culture in a sustainable manner. This study highlighted the importance of including mental health education in teacher training programs. Educators should be given resources on teaching students how to best face the real world and overcome obstacles to improve their mental health. This empirical study used both qualitative and quantitative methods to analyze Zhuangzi's concept of self-adaptation. The findings can be applied to improve student mental health, but they only serve as a starting point. It is hoped that more academics from different fields will pay attention to this issue.

The current trend in academia is towards cross-field research and passing on cultural heritage education. This study can serve as a bellwether on how traditional cultures inspire contemporary lives and help the general public learn techniques to support mental health through Zhuangzi. It is also a good reference for issues related to the philosophy of life education. No one can avoid frustrations. Even Thomas Edison said, "Genius is 1 percent inspiration and 99 percent perspiration.". Without a healthy mind to help deal with various frustrations, one's chances of achieving a specific goal are diminished. Unfortunately, with the contemporary culture full of strong competition, many students struggle with overcoming challenges, and the current education system should pay more attention to this issue.

While standing on a bridge, Zhuangzi saw some fish swimming in the water. When he described how happy the fish were, Huizi asked Zhuangzi how he could know whether the fish were happy. This debate is the most famous conversation from the Zhuangzi. This study analyzed Zhuangzi's concept of self-adaptation and his empathy towards the fish, and the results of this analysis represent 
the academic research findings of this study. On the one hand, teachers can use this study as a basis for the interpretation of this debate. On the other hand, Zhuangzi's understanding of self-adaptation can be expanded to cover mental health education in the sphere of life education or affective education in order to teach students how to maintain a peaceful mindset and healthy values in the face frustration or depression. Zhuangzi's observations about the fish in the water are related in spirit to the study of science. His empathetic inference that the fish were happy is related in spirit to the study of philosophy. The addition of educational morals to the symbolic meaning of the fish's happiness in the water is related in spirit to the study of education. Thus, the research methods section covered how the study represents the disciplines of philosophy, education, and science. The researcher believes that these three fields can not only complement each other but also enhance the influence of life philosophy education due to the cross-field spirit.

This study is a work of exploratory research. Future researchers can use the 11 self-adaptation categories identified in this study as a starting point to explore regular events in society that may have negative influences on mental health. Such research could also analyze how Zhuangzi's concept of self-adaptation impacts mental health. The author believes that as modern education continues to pursue creative thinking and learning without limitations, teachers should go beyond the limitations of their professional fields by integrating more mental health education into their curricula. Educators play an important role in cultural inheritance and innovation. Teachers are the driving force behind a sustainable, healthy social and cultural climate, and they support this type of environment by paying attention to sustainable methods of encouraging student mental health. Thus, this study proposed extending and expanding current approaches to university students' mental health issues. In other words, the core value of this study's contributions to modern education lies in empowering teachers. This study gives educators more tools to help students with their mental health by improving their abilities to make adjustments when facing frustrating situations so that they can build healthy and active lives with regard to their careers and other facets of life.

Furthermore, a class is a small social system, and it is important to acknowledge a teacher's role in such an environment. How can teachers push their students to grow in their education-related interactions without damaging a student's self-respect and having a negative influence on student mental health? In the process of education, a student who suffers from mental trauma may develop an antisocial personality. Therefore, teachers must continue to hone their abilities to mentor students without harming their mental health. Thus, based on the concept of symbolic interactionism, this study suggests that researchers position their studies in modern education to pay particular attention to supporting a creative learning environment. Under the premise of using teaching models that are different from traditional ones, researchers can further explore factors behind the relationship between a teacher's role and student mental health by looking, for example, at teacher-related linguistic and non-linguistic factors. Researchers can also conduct empirical studies on the positive and negative influences on student mental health in response to the continuing development of educational dynamics in this era.

Since the beginning of human history, from ancient civilization to modern times, there have been many thoughts on the philosophy of life. Philosophical theories related to identifying problems and finding solutions are all built on the core value of what it means to be human. Although throughout the history of human development there have been a variety of living conditions and lifestyles, the essence of being human has always been the same. Therefore, philosophies of life will never lose their value even as times change. In the modern digital age, the burdens of learning, living, and working are heavier than ever. Students must take multiple assessments when it comes to schooling and the cultivation of professional skills. If students lack the courage to face their frustrations and healthy mental states are neglected, then young people are likely to suffer setbacks that can lead them to never become productive members of society. Therefore, more attention should be paid to cultivating Zhuangzi's concept of self-adaptation in modern education. It was hoped that this study will make 
positive contributions to mental health education and that the findings will serve as a call to direct more attention in the education system to student mental health issues.

Focus on improving student mental health in current education systems remains limited. As such, all educational disciplines must work together to make improvements. This study calls on educators to design relevant courses to provide students plenty of ways to improve their mental health. Only by doing so can educators ensure that young people's talents, potential, and genius are being put to good use. In the field of philosophy, ancient Eastern thinkers had similar belief to their Western counterparts. For example, Socrates's idea that knowledge is a virtue and Confucius's belief in extending knowledge and cultivating oneself both support the concept of improving mental health through education. This study expects that the research findings can actually be applied to modern education to improve student mental health and bring more happiness to society and culture. With the higher levels of pressure associated with the age of digital technology, educators and education leaders hold the responsibility of teaching students how to deal with life's frustrations.

It is suggested that Chinese curricula and life philosophy lessons can be designed based on this study's findings using the concept of self-adaptation as explained by Zhuangzi. With a teacher's guidance, students can learn self-adaptation by following these steps: (a) Share their individual frustrating experiences and (b) apply the concept of self-adaptation to them in order to better cope with life's troubles. When performing these steps, students must do two things: (a) Compare their feelings about their frustrations before applying the idea of self-adaptation to their feelings after the concept has been applied and (b) verify that the psychological energy of positive thinking has increased within them as individuals. Since the above example is a simple framework, it can be applied easily and broadly. The philosophy of self-adaptation, if explored and applied correctly by students, should yield positive outcomes in their lives. The current trend in academia is towards cross-field research and passing on cultural heritage through education. This study can serve as a bellwether on how traditional cultures inspire contemporary lives and help the general public learn techniques to support mental health through Zhuangzi. It is also a good reference for issues related to the philosophy of life education. No one can avoid frustrations. Even Thomas Edison said, "Genius is 1 percent inspiration and 99 percent perspiration.". Without a healthy mind to help deal with various frustrations, one's chances of achieving a specific goal are diminished. Unfortunately, with the contemporary culture full of strong competition, many students struggle with overcoming challenges, and the current education system should pay more attention to this issue.

Funding: This research received no external funding.

Conflicts of Interest: The authors declare no conflict of interest.

\section{References}

1. Shen, L. Affective education. Taiwan Educ. 2005, 636, 2-12. [CrossRef]

2. Lin, A.W. A research on the theoretical foundation and practicality of affectional education in colleges. A Reexamination on Theories \& Realization Based on Confucianism, Taoism and Buddhism. Gen. Educ. Q. 1998, 5, 13-26. [CrossRef]

3. Shen, V. On the development of human affectivity and practical wisdom. J. Gen. Educ. 1998, 5, 65-84. [CrossRef]

4. Fong, T.L. The religiositaet and ecological thinking. Theory Emot. Educ. 1998, 5, 85-102. [CrossRef]

5. Yu, A. Philosophical counseling as ethical practice. Philos. Cult. 2010, 37, 85-108. [CrossRef]

6. Gong, J.B. The Rise of Contemporary Taoist. Available online: http://www.etaoist.org/taoist/index.php/201108-22-02-11-46/2011-09-13-16-05-29/1245-2011-10-23-03-19-28 (accessed on 1 December 2018).

7. Lee, S.H. Inquiry on Zhu Xi's theory of "transforming the temperament". Taiwan J. East Asian Stud. 2010, 7, 129-153. [CrossRef]

8. Raabe, P.B. Healing words: Philosophy in the treatment of mental illness. Philos. Cult. 2010, 37, 21-34. [CrossRef] 
9. Shu, K.Y. A brief study on the applications of the Taoist philosophy in students' work. Sci. Technol. 2017, 20, 352.

10. Zheng, T.X. German Taoist Studies: Leibniz Praises Taoist Culture. Available online: http://rufodao.qq.com/ a/20150803/035471.htm (accessed on 11 November 2018).

11. The China Press. The American Laozi Institute was Established in New York. Available online: http: //ny.uschinapress.com/spotlight/2017/10-06/129901.html (accessed on 1 December 2018).

12. Tsai, C.M. A Study on the General Public's Reception of Taoism Based on the Modern Popular Taoist Books in the US. Available online: http://blog.sina.com.cn/s/blog_755ee2620102yb9u.html (accessed on 8 January 2018).

13. Liberty Times Net. The Moment of a Female Japanese High School Student Jumping in Front of a Moving Train Taken from Her Own Live Streaming. Available online: http://news.ltn.com.tw/news/world/breakingnews/ 2475700 (accessed on 3 July 2018).

14. Newtalk.tw. The Suicide Trend: The Suicide Rate Has Increased by $60 \%$ over the 45 Years. Available online: https://newtalk.tw/news/view/2016-04-27/72599 (accessed on 1 December 2017).

15. Lin, H.J. A study on the adaptive teaching and counseling from the philosophical perspective: The secularity and the sacredness of education. Second. Educ. 2016, 67, 3-17. [CrossRef]

16. Chang, M.H. Philosophy is everywhere in life: Hao-Cheng Fu illuminates logical thinking. Eval. Bimon. 2018, 73, 32-34.

17. Li, B. Philosophical counseling and humanities therapy. Philos. Cult. 2017, 44, 5-19.

18. Li, W.T. An exploration of Mencius and Zhuangzi's view of life and death. Philos. Cult. 2018, 45, 147-160.

19. Chen, S.T. Positioning mind in the world: Scientific practice in context and its implication for other practices. J. Taiwan Univ. Lit. Hist. 2016, 85, 219-254. [CrossRef]

20. Shen, V. Body, metaphor and dialogue: Daoist spirituality and discourse from Zhuangzi to Laozi middle scripture. Philos. Cult. 2018, 45, 125-144.

21. Huang, Y.L. A discussion about "body": A formless being, informed by "Grand Master Zhuang Ze". Philos. Cult. 2018, 45, 21-39.

22. Obert, M. A discussion of reification through participation in the Zhuang $\mathrm{Zi}$ from the stance of responsiveness in Emmanuel Levinas and the Japanese Stone Garden. J. Taiwan Univ. Lit. Hist. 2017, 87, 151-178. [CrossRef]

23. Cheng, Z.Y. Exploring Zhuangzi's concept of meliorism by examining Dai Junren's "Interpretation of the Happiness of Fish". J. Taiwan Univ. Lit. Hist. 2017, 88, 1-31. [CrossRef]

24. Huang, Z.H. The therapeutic implications of the consummation of life in the chapter of "On Equalizing Things". Legein Mon. 2017, 509, 23-38.

25. Huang, W.H. On the problem of "transcendence" in phenomenology: In view of Nishida's philosophy. J. Taiwan Univ. Lit. Hist. 2016, 84, 143-172. [CrossRef]

26. Li, M. A Pandect Chapter Commentaries of Zhuangzi; The Commercial Press (CPTW): Taipei City, Taiwan, 1990; ISBN 9570502088.

27. Lyan, L.L. Analysis on "Mountain Wood" of Zhuangzi. J. Natl. Huwei Univ. Sci. Technol. 2009, 28, 27-42.

28. Chyou, W.T. The rebuilding of recognition: The study of Zhuangzi "Chi Wu Luan". J. Grad. Inst. Chin. Lit. Fu Jen Cathol. Univ. 2001, 11, 1-17. [CrossRef]

29. Hwang, Y.J. A study on Zhuangzi's state of wandering based on "Carefree Wandering". J. Grad. Inst. Chin. Lit. Fu Jen Cathol. Univ. 2000, 10, 69-89.

30. Lin, M.J. Watching, reflection and concentration: Visuality in the philosophy of Zhuangzi. Chin. Stud. 2012, 30,1-33. [CrossRef]

31. Syu, D.S. A study on the nature theory of Zhuangzi. Huafan J. Humanit. 2008, 10, 37-86. [CrossRef]

32. Yuan, S.A. Agreement and tolerance: A study on Zhuangzi's thought on tolerance. Mon. Rev. Philos. Cult. 2000, 27, 27-36. [CrossRef]

33. Lyou, S. The characteristics of Zhuangzi's philosophic thoughts. J. Educ. Sci. Hunan Norm. Univ. 2004, 33, 5-7.

34. Lyou, C.G. The principal part of the spirit in Zhuangzi. Res. Dev. 2006, 13, 97-107.

35. Syu, Y.C. A new research of Zhuangzi's philosophy of travel-combine theory with practice. Mon. Rev. Philos. Cult. 2015, 42, 169-186.

36. Lyu, W.J. Preliminary understanding health care concept of Zhuangzi. Taipei J. Tradit. Chin. Med. 2007, 10, 55-64. 
37. Shen, W.H. The great use of uselessness: Study on the thought of Zhuangzi. Chin. J. 2014, 29, 167-186.

38. Shen, W.H. The enjoyment of fish: A debate between Zhuangzi and Hwei-Shr. Chin. J. 2013, 27, 137-154.

39. Chen, Y.W. A discussion on the theory of ruthlessness in Zhuangzi. Annu. Grad. Sch. Chin. Lit. Soochow Univ. 2016, 12, 185-196.

40. Li, T.W. Zhuangzi's comprehension and transmission of Dao and his vision of free and easy wandering. Legein Mon. 2016, 498, 3-15.

41. Lin, B.H. Zhuangzi interpreting the meaning of "tours". J. Inst. Chin. Lit. Shih Hsin Univ. 2008, 4, 79-105. [CrossRef]

42. Sye, M.Y. The systematic thought behind Zhuangzi's theory of qi. Legein Mon. 1998, 279, 18-24. [CrossRef]

43. Wang, J.M. The connotation of use of Zhuangzi. J. Chin. Lit. Natl. Dong Hwa Univ. 2006, 1, 45-66. [CrossRef]

44. Deng, K.M. Fang Yi-Jr on "Zhuangzi": Focusing on Tao and Object. Chin. Stud. 2013, 31, 1-29.

45. Jyang, Y.Y. Ju-Syi's review on Zhuangzi. Zhongji Xuekan 2002, 2, 91-116. [CrossRef]

46. Lin, M.J. Other titles: Non-self and all is self Lyu Hwei-Ching's thought about non-self in commentary on Zhuangzi. Stud. Sinol. 2013, 35, 1-32. [CrossRef]

47. Lai, S.S. Natural change and metaphors of life and death in Zhuangzi. Chin. Stud. 2001, 29, 1-34. [CrossRef]

48. Wu, C.H. The view on life and death in Zhuangzi. Annu. Grad. Sch. Chin. Lit. Soochow Univ. 2010, 6, 121-140. [CrossRef]

49. Tsai, B.M. The expert's "sense of the body" in Zhuangzi: Developing a new field of research in Daoism. Chin. Stud. 2015, 33, 73-108.

50. Lyou, T.L. Body, metaphor and the power of transformation: On two kinds of body and thinking in Zhuangzi. Tsing Hua J. Chin. Stud. 2014, 44, 185-213. [CrossRef]

51. Jung, J.Y. The phenomenology of qi by Zhuangzi. Bull. Inst. Chin. Lit. Philos. 2013, 42, 109-148.

52. Lyou, J.W. Sense and the Tao: The perception of communication by Zhuangzi. Chung Cheng Chin. Stud. 2015, 26, 73-93.

53. Syao, Y.M. From heart to Tao and then to the world: A study on the fundamental structure of the thoughts in Zhuangzi. Sun Yat-sen J. Humanit. 2006, 23, 45-74. [CrossRef]

54. Chen, D.H. The state of the Tao attitude in Zhuangzi's philosophy. Legein Soc. 2000, 24, 41-72. [CrossRef]

55. Lin, S.L. The relationship between Zhuangzi and study of Confucian classics. Hungkuang Acad. Rev. 1999, 34, 387-411. [CrossRef]

56. Tung, H.P. Zhuangzi and the Mohist School. Acad. J. Zhongzhou 2004, 5, 162-165.

57. Jwang, W.S. Zhuangzi and the Yin-Yang School. J. Natl. Taiwan Norm. Univ. Humanit. Soc. Sci. 2000, 45, 1-13. [CrossRef]

58. Swun, D.L. An appreciation of Zhuangzi's esthetic conception. J. Shenyang Norm. Univ. Soc. Sci. 2005, 29, 52-55.

59. Syao, J.B. Is there aesthetics in Zhuangzi? Reconstruction of the aesthetics in Zhuangzi. Legein Soc. 2011, 47, 1-40. [CrossRef]

60. Wang, J.Y. The theory of emptiness and stillness and literary works of Zhuangzi. Orient. Humanit. 2007, 6, 31-37. [CrossRef]

61. Neu, W.Y. Qualitative Research Methods and Dissertation Writing, 2nd ed.; Yeh Yeh Book Gallery: Taipei City, Taiwan, 2018.

62. Han, C. Research Method Theories, 2nd ed.; Wunan: Taipei City, Taiwan, 2012.

63. Yen, Y.S. (Ed.) Eurasia's Modern Practical English-English and English-Chinese Dictionary; Chuang-Yuan Publication: Taipei City, Taiwan, 1979.

64. Zhang, F.J. (Ed.) International English-Chinese Dictionary; Eurasia: Taipei City, Taiwan, 1988.

65. Yen, Y.S. (Ed.) Eurasia's the Most Up-Date English-Chinese Dictionary; Eurasia: Taipei City, Taiwan, 1988.

66. Yongda Editing Committee (Ed.) A New Practical English-Chinese Dictionary; Yong Da: Taipei City, Taiwan, 1990.

67. Yen, Y.S. (Ed.) Time English-English and English-Chinese Dictionary; Wan Ren: Taipei City, Taiwan, 2001.

68. Wang, W.K. Educational Research Methodology; Wunan: Taipei City, Taiwan, 2001.

69. Online Chinese Dictionary. zi (Chinese Pinyin). Available online: http://dict.revised.moe.edu.tw/cgi-bin/ cbdic/gsweb.cgi?o=dcbdic\&searchid=W00000009443 (accessed on 21 February 2019).

70. Online Chinese Dictionary. shi (Chinese Pinyin). Available online: http:/dict.revised.moe.edu.tw/cgi-bin/ cbdic/gsweb.cgi?o=dcbdic\&searchid=W00000008795 (accessed on 3 July 2019). 
71. Online Chinese Dictionary. zi shi (Chinese Pinyin). Available online: http://dict.revised.moe.edu.tw/cgi-bin/ cbdic/gsweb.cgi?o=dcbdic\&searchid=Z00000137888 (accessed on 21 February 2019).

72. Lin, Y. Chinese-English Dictionary of Modern Usage; The Chinese University of Hong Kong: Hong Kong, China, 1972.

73. Liang, S.C. Far East Chinese English Dictionary; Far East Books: Taipei City, Taiwan, 1992.

74. Chang, S.S. Research Methods, revised version; Tsang Hai Book Publishing Co: Taichung, Taiwan, 2001.

75. Wu, M.C. Educational Research: Basic Concepts and Method Analyses; Wunan: Taipei City, Taiwan, 1996.

76. Kao, J.W. Qualitative Research Methods; Shita Books: Taipei, Taiwan, 2002.

77. Popkewitz, T.S. Paradigms in educational science: Different meanings and purpose to theory. J. Educ. 1980, 162, 28-46. [CrossRef]

78. Dai, J.Y. Statistics: Principles and Applications; Tsang Hai Book Publishing Co: Taichung, Taiwan, 2004.

79. Chung, L.N. Applied Social Science Research Methods; The Commercial Press: Taipei City, Taiwan, 1998.

80. Chang, F.F.; Tang, G.D. Survey Research; Chia, F.M., Yang, S.K., Eds.; Shita Books: Taipei City, Taiwan, 1992; pp. 35-68.

81. Chen, B.C. Educational Thoughts and Educational Research; Shita Books: Taipei City, Taiwan, 1988.

82. Huang, G.H.; Chien, M.F. Educational Research Methods; Shita Books: Taipei City, Taiwan, 1995.

83. Wang, W.K.; Wang, C.H. Educational Research Methods; Wunan: Taipei City, Taiwan, 2014.

84. Lin, S.C. Educational Research Methods: All-Directional Summary and Analyses; Psy Books: Taipei City, Taiwan, 2003.

85. Lin, C.W. Statistics: Applications in Education and Counselling; Yeh Yeh Book Gallery: Taipei City, Taiwan, 2008.

86. Guo, S.Y. Psychological and Educational Research Methods; Jing Hwa: New Taipei City, Taiwan, 2009.

87. Yeh, C.S. Educational Research Methods; Psy Books: Taipei City, Taiwan, 2001.

88. Chu, C.T. Sampling Methods; Sanmin: Taipei City, Taiwan, 1993.

89. Niu, W.Y. Qualitative Research Methods and Essay Writing; Yeh Yeh Book Gallery: Taipei City, Taiwan, 2018.

90. Chen, H.M. The Qualitative Research in Social Science; Wunan: Taipei City, Taiwan, 2006.

91. Wang, W.K. Qualitative Educational Research Methods; Shita Books: Taipei City, Taiwan, 1994.

92. Tsai, Y.M.; Liao, P.S.; Lin, N. Sociology Research Methods; Tonsan: Taipei City, Taiwan, 2007.

93. Chiu, H.C. Quantitative Research Methods (I): Research Design and Data Processing; Yeh Yeh Book Gallery: Taipei City, Taiwan, 2008.

94. Feng, C. Statistics; CTS Culture: Taipei City, Taiwan, 1989.

95. Liu, H.H. Social Statistics; Yeh Yeh Book Gallery: Taipei City, Taiwan, 2003.

96. Huang, C.J. The principles and methods of qualitative research. In Qualitative Educational Research: Methods and Examples; Honman: Taipei City, Taiwan, 1998.

97. World Health Organization. Depression and Other Common Mental Disorders: Global Health Estimates. Resource Booklet 2017. Available online: http://www.who.int/mental_health/management/depression/ prevalence_global_health_estimates/en/ (accessed on 23 December 2017).

98. For More Information on the Prevalence of depression across the World, See the World Health Organization's Publication, "Depression and Other Common Mental Disorders: Global Health Estimates.". Available online: http://www.who.int/mental_health/management/depression/prevalence_global_health_estimates/en/ (accessed on 1 August 2018).

99. Hidaka, B.H. Another informative reference on the topic of depression is Hidaka's study, which seeks to explain the increasing prevalence of depression. Depression as a disease of modernity: Explanations for increasing prevalence. J. Affect. Disord. 2012, 140, 205-214. [CrossRef]

(C) 2019 by the author. Licensee MDPI, Basel, Switzerland. This article is an open access article distributed under the terms and conditions of the Creative Commons Attribution (CC BY) license (http://creativecommons.org/licenses/by/4.0/). 